\title{
Quasi-periodic Geometry for Architectural Acoustics
}

\author{
Rima Ajlouni
}

\begin{abstract}
The discovery of quasi-periodic atomic order in the crystalline state has uncovered an exciting new class of symmetries that has never been explored. Quasi-periodic structures offer unique opportunities for investigating questions related to their acoustical behavior because of their non-periodic translational order and self-similar properties. Their unique long-range non-periodic formations have the ability to diffuse and orchestrate the flow of sound energy in many unique ways; offering intriguing potential for innovating a new wave of optimized sound diffusers. One key limitation with available periodic diffusers is that their repeating logic creates repetitive energy loops, which significantly reduce their ability to uniformly disperse sound energy. Quasi-periodic geometry can mitigate such limitation. By encapsulating an infinite variety of distinct profiles in all directions, quasi-periodic surfaces can eliminate the formation of bundled or looped reflections; considerably enhancing the ability of the diffuser to uniformly disperse sound energy. To investigate this hypothesis, the diffusion quality of a quasi-periodic surface is compared to the diffusion performance of Schroeder's 2D Quadratic Residue Diffuser. The normalized diffusion coefficients and polar response plots were calculated for both surfaces. Results show that the diffusion quality of the tested quasi-periodic surface is superior to the diffusion performance of the 2D Quadratic Residue Diffuser.
\end{abstract}

Keywords: Architecture acoustics, Diffusion quality, Quasi-periodic surfaces, Schroeder's Quadratic Residue Diffuser.

\subsection{INTRODUCTION}

The discovery of quasicrystals in the 1980s has uncovered an exciting new class of geometric structures that was not known before (Shechtman et al. 1984). The atomic arrangements of this new state of matter exhibit a global long-range non-periodic translational order, which was thought to be forbidden for the crystalline state in the traditional framework of crystallography. These formations can fill the space ad infinitum without repeating the same formations (Levine and Steinhardt 1984; Socolar, Steinhardt and Levine 1985; Levine and Steinhardt 1986; Ishii and Fujiwara 2008). One key ingredient of the quasi-periodic order is the multi-level hierarchical nature, which allows the same patterns to recur at multiple scales. Three decades after their initial discovery, hundreds of quasicrystals have been documented; exposing a wealth of quasi-periodic symmetries with untapped potentials (Jazbec 2009; Maclá 2006; Yamamoto and Takakura 2008; Dubois 2012).

Stable URL: https://arcc-journal.org/index.php/arccjournal/article/view/453 DOI: 10.17831/enq:arcc.v15i1.453

Corresponding Author: ajlouni@arch.utah.edu

Published by the Architectural Research Centers Consortium under the terms of the Attribution-NonCommercial-ShareAlike 4.0 International license 
Aside from their astonishing visual and physical qualities, quasi-periodic formations exhibit unusual structural properties, which can be ideally suited for investigating new applications in architecture and design. These systems could potentially provide a rich source of performative geometries for innovating new structures, systems, materials, patterns, surfaces and forms. One intriguing potential of their unique quasi-periodic formations is the ability to orchestrate the flow of light or sound energy, which could find applications across a wide range of light and soundbased technologies (Roichman and Grier 2005; Steurer and Widmer 2007; Mikhael et al. 2010; Schmiedeberg and Stark 2012; Martinsons et al. 2014; Dong et al. 2015; Boriskina 2015; Schmiedeberg et al. 2017). In this context, this paper is focused specifically on investigating aspects of the acoustical diffusion behavior of quasi-periodic formations.

One key challenge associated with designing effective surface diffusers is the ability to disperse sound energy uniformly over the diffused sound field while avoiding the formation of orchestrated, bundled or looped reflections (D'Antonio and Cox 2004, $2009,2017)$. It is assumed that better diffusion occurs when every position in the diffuse sound field receives the same density of the reflected energy (D'Antonio and Cox 2004, 2009, 2017; Fay 2013; Kleiner and Tichy 2014). However, it is a common practice to design diffusers based on periodic repetition of a limited number of basic shapes, which often creates repetitive energy loops; significantly reducing the ability of the diffuser to uniformly disperse sound energy (Lam 1999; D'Antonio and Cox 2004; Cox and D'Antonio 2009; Hughes et al. 2010). To mitigate the periodicity problem some researchers turned their attention to investigate random or pseudo-random designs (Angus 1995; Hughes et al. 2010). While this approach can eliminate periodicity, random arrangements are hard to visually encode or predict and often requires the manufacturing of the large number of shapes which make them costs prohibitive (Cox and D'Antonio 2004). Other studies used modulation schemes of one or two basic shapes and relied on visual appearance, random sequences, number theory, or optimization programs to arrange the units in a non-repeating manner (Angus 1995, D'Antonio and Cox 2004; Cox and D'Antonio 2009). While such schemes can optimize the number of basic shapes, their pseudo-random logic is often dictated by the shape of the basic units (i.e. squares, triangles or hexagons) which can limit the range of possible arrangements and design variations. Moreover, these geometric designs are rigid and their visual appearance is often ignored in the pursuit of more functional attributes; making them unattractive options for architectural environments. Another modulation approach utilizes Fibonacci onedimensional quasi-periodic sequence to arrange the basic repeating units (Arau-Puchades 2016). However, these arrangements exhibit quasiperiodicity only in one direction and their design choices and aesthetics can be very limited. Recently, Lee and colleagues (Lee, Tsuchiya and Sakuma 2018) investigated variations of surface profiles designed by rearranging the thick and thin rhombuses in Penrose tiling. After comparing tile arrangements (symmetric, asymmetric and random), they found that the effect was relatively small when comparing Penrose tiling with random tiling. This suggest that quasi-periodic formations have advantages over periodic formations. However, their study did not compare the performance of Penrose-type surfaces with available diffusers.

Based on this review, the ideal scheme for designing surface diffusers requires that periodicity be removed from all directions and using a limited number of manufactured shapes while at the same time allowing for a wider space for creativity and design aesthetics. This paper argues that three-dimensional quasiperiodic structures offer unique opportunities for satisfying these requirements. In addition, their selfsimilarity provides opportunities for designers to include detailed profiles at multiple scales for optimizing performance (Cox and D'Antonio 1997; D'Antonio and Konnert 1992; Cox and D'Antonio 2004, 2009,2017; Qian 2001; Bradley et al. 2011; Ning and Zhao 2017; Ajlouni 2017a).

Today, a major roadblock is challenging designers to fully engage in investigating the use of quasi-periodic formations for acoustical surface designs, which is associated with the difficulties to generate the complicated quasi-periodic formations. Unfortunately, most of the available structural models are based on complicated or abstract mathematics (i.e. matching, substitution, inflation, deflation, matching, projection, etc.) (Penrose 1974; De Bruijn 1981a; De Bruijn 1981b; De Bruijn 1981c; Bak 1986; Socolar, Steinhardt and Levine 1985; Lord, Ranganathan and Kulkarni 2000; Abe, Yan and Pennycook 2004; Madison 2015a; Madison, 2015b), which present substantial limitations for researchers especially in the non-technical fields. Fortunately, the 
discovery of historical patterns exhibiting quasiperiodic formations is providing new insights into understanding these complicated structures (Makovicky 1992; Makovicky, P'erez and Hach-Al'ı 1998; Rigby 2005; Lu, and Steinhardt 2007; Saltzman 2008; Makovicky and Makovicky 2011). Derived from these traditional principles, a simple proportional structural model for generating these complicated formations has been described (Ajlouni2012; Ajlouni2013; Ajlouni2017b; Ajlouni2018). This paper utilizes this new approach to generate the quasiperiodic surface design tested by this research.

\subsection{BACKGROUND}

In the context of architectural design, the challenges associated with the acoustical prediction can be attributed to the interplay of a complex system of competing factors including, space size, proportion, geometry, material properties and surface details. One of the critical drivers of acoustical behavior relates to the design of architectural surfaces, which primarily affect the way in which sound reflections propagate through space. If such reflections are not carefully steered, they can create strong secondary sound patterns; often causing flutter echoes ${ }^{1}$ or comb filtering ${ }^{2}$ (Cox and D'Antonio 2009). With proper acoustical design of surface geometry, sound reflections can be effectively tamed by evenly diffusing the reflected sound energy so it is less focused or coherent (Fay 2013). The behavior of sound reflections off different surface geometry and their effect on shaping the spatial auditory atmosphere have been investigated in many architectural contexts (Cox and Lam 1993; Cox 1996; Embrechts, Archambeau and Stan 2001; Funkhouser et al. 2002). Today, a limited range of contemporary sound diffusers are designed with certain geometric profiles to either scatter the sound spatially, temporally or both (Farner 2014). However, depending on their surface designs, arrangements and depth profiles, the performance of sound diffusers is often limited by their ability to respond to the targeted sound wavelength (Everest and Pohlmann 2009; Bradley et al. 2011). Only a limited number of available diffusers are designed to effectively respond to a range of wavelengths. Some of the most functional diffusers were invented in 1970s by Manfred Schroeder, who used number theory to predict the optimal diffusion for a surface profile (Schroeder 1975; Schroeder 1979). With the introduction of Schroeder's Quadratic Residue Diffusers (QRD), it was possible for the first time to measure the complete diffuse reflection based on the grating loops produced by the periodic phase grating energy (Cox et al. 2006). Schroeder's diffusers are designed with a sequence of wells of the same width and different depths and can be arranged in one or multi-dimensional devices (Cox and D'Antonio 2004). Since their introduction, Schroeder's diffusers have been widely adopted in technical and architectural acoustics (Cox and D'Antonio 2004, 2009, 2017; D'Antonio and Cox 2000; Cox and Lam 1994; Hargreaves, Cox and Lam 2000). However, in terms of repeating the optimized sequence "the curse of periodicity" presents a major limitation (Cox and D'Antonio 2004, 248). Moreover, at low frequencies, Schroeder diffusers can cause high absorption due to the resonance created in the deep well system (Fujiwara 1995). Aesthetically, the visual appearance of Schroeder's irregular well arrangements can be challenging for designers (Zhu et al. 2017). Their protruding profiles make them unattractive options for architectural environments. Today, the lack of new designs that can complement contemporary architecture presents a major challenge (Cox et al. 2006). Only a limited number of available diffusers are considered acceptable for architectural spaces. Computer calculations are often utilized to numerically design and optimize these surfaces (Cox and D'antonio 2009; Lee and Sakuma 2015; Henham, Holloway and Panton 2016). However, such design process is limited to the manipulation of some basic design elements and is often time intensive, as it evaluates unit by unit basis. With the limited availability of aesthetically acceptable diffusers for architectural acoustics, it is critical that new and original approaches are proposed. The need for innovative designs that can satisfy both the acoustic and visual requirements is therefore essential.

\footnotetext{
${ }^{1}$ Sound vibrations are caused when energy is trapped within a reflection cycle between two surfaces.
}

2 The amplification of certain sound frequencies while reducing others. 


\subsection{METHODS}

\subsection{Research Design}

The aim of this research is to explore the potential of using quasi-periodic formations to design new surface diffusers. The hypothesis argues that because of their non-periodic translational nature, quasi-periodic surfaces offer many advantages over periodic designs in terms of eliminating the creation of repetitive energy loops and enhancing the ability of the diffuser to uniformly disperse sound energy. In this research, a new type of quasi-periodic surface diffuser based on 2D Penrose tiling formations is developed and tested. To establish a base-performance criterion for comparison, Schroeder's 2D Quadratic Residue Diffuser (QRD) with similar depth profile is also tested. The ISO standard metrics (Normalized Diffusion Coefficient and Spatial Response Plots) are used to measure the quality of the diffuse reflection caused by both surfaces. These metrics are used to evaluate the quality of the diffusers in terms of the spatial uniformity of the reflected sound energy (Cox et al. 2006). In particular, the Normalized Diffusion Coefficient provides a measure of the spatial distribution of the reflected sound energy and it was developed with the primary goal of defining an accurate ISO standard metrics for evaluating the worth of surface diffusers. It can be used as a tool for ranking the quality of different diffusers and is calculated as a single value between 0 and 1 , which measures the uniformity of the reflected energy. The value of 1 indicates that the reflected energy is evenly distributed across all directions, while a value of 0 indicates that the reflected energy is reflected only in one direction.

\subsection{Instrumentation}

The two-dimensional acoustics simulation software AFMG Reflex was used to model the diffusion of sound wave incident upon the defined surface profiles. The numerical method calculates the diffusion properties of the sample surfaces based on the Boundary Element Method (BEM). The diffusion coefficients are plotted as frequency response graphs and the reflective properties are displayed as a polar response graph for a specific angle of incidence and frequency of a sound wave (AFMG 2018).

\subsection{Target population}

For the quasi-periodic surface designs, the general target population includes all categories of quasiperiodic symmetries including 5-fold, 7-fold, 8-fold, 9-fold, 10 -fold and 12-fold. However, for the purpose of conducting this research, numerical measurements specifically target 5 -fold three-dimensional quasiperiodic structures, with the assumption that it provides a true representative sample of the general quasi-periodic population.

For the periodic surface design, Schroeder's 2D Quadratic Residue Diffuser (QRD) is used to establish a base for comparison with the assumption that it provides a true representative sample of the general periodic population. This is a two-plane diffuser formed by arranging series of wells in two directions which generate diffuse reflections in threedimensional (3D) space. In this paper, a sequence array of $7 X 7$ quadratic residue diffuser is used as the basic unit for the periodic arrangement.

\subsection{Generating sample profiles 3.4.1: Quasi-periodic geometry}

The number of the quasi-periodic designs that can be generated based on five-fold rotational symmetry can be extensive depending on the underlying structural order and the internal geometry. For the purpose of this research, three-dimensional quasi-periodic surface profiles are generated based on 5 -fold quasiperiodic Penrose tiling using the thin and thick rhombus (Figures 1) (Penrose 1974). While this research focuses specifically on Penrose designs, the underlying goal is to develop a general method for testing other types of quasi-periodic structures.

Penrose tiling is a non-periodic tiling discovered by mathematical physicist Roger Penrose in the 1970s (Penrose 1974). In a five-fold rotational symmetry, Penrose discovered that by using only two tiles constructed based on the irrational number Phi $(\phi=$ $1.618033988749895)$, a surface can be tiled infinitely without repeating the same formations (Figure1). Using the thin and thick rhombus, the infinite quasiperiodic Penrose tiling can be assembled according to very specific local matching rules (Figures1 and 2). 

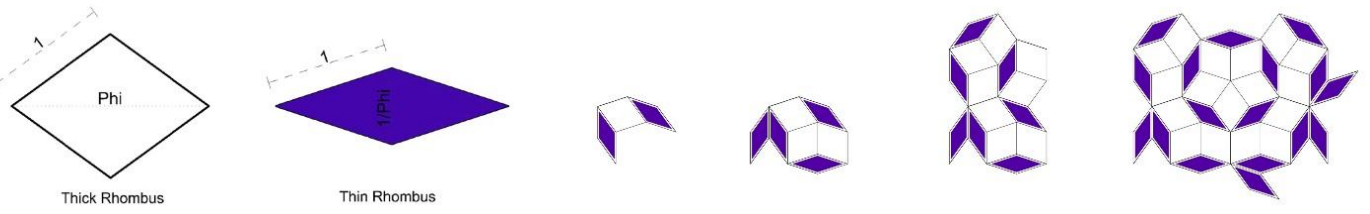

Figure 1 - Using the thin and thick rhombus, which are constructed based on Phi, quasi-periodic Penrose tiling can be assembled according to specific local matching rules.

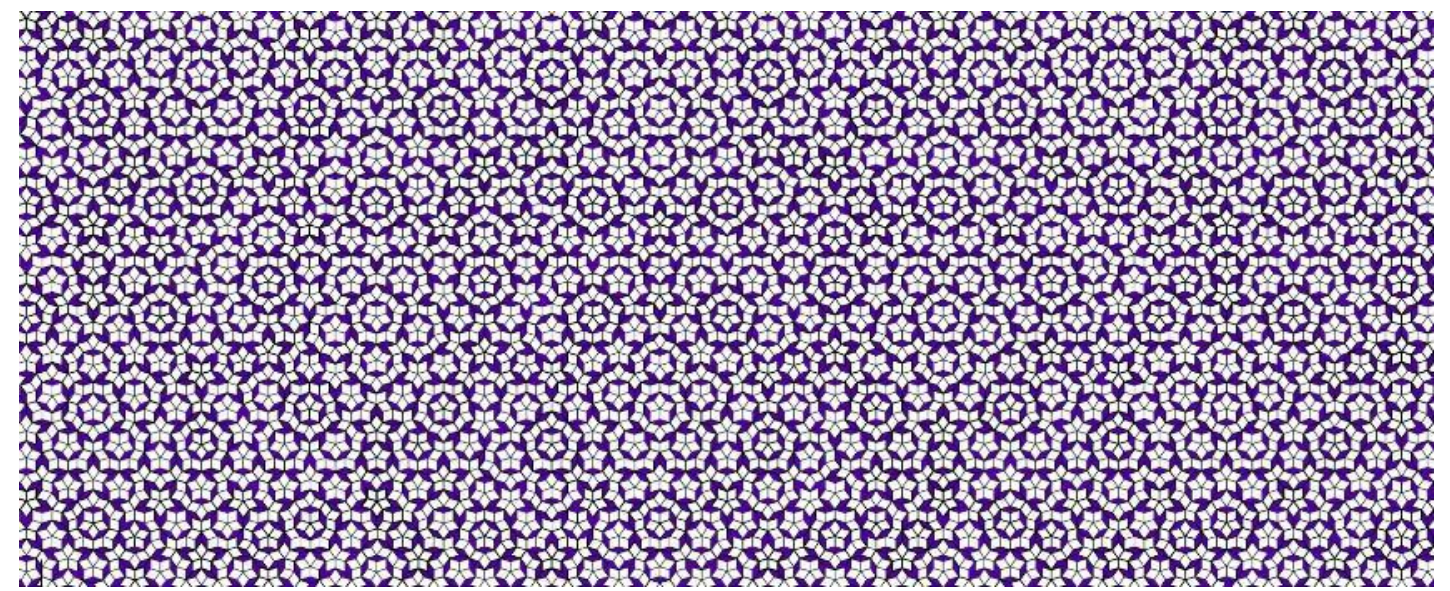

Figure 2 - An extended formations of Penrose quasi-periodic tiling using the thin and thick rhombus.

The construction process of 5 -fold Penrose tiling pattern is governed by a proportional system that is derived from the traditional method of using a compass and a straightedge (Ajlouni2011). A framework of nested decagrams serves as the underlying hidden grid for guiding the construction process of the quasi-periodic system (Figure 3). The infinite Penrose empire is constructed by building a progression of nested sequences, in which, each decagram is built to the previous level. This framework is constructed by drawing a polar array of lines through connecting points of equal distances on the initial decagon. The framework grows based on the Golden ratio. If we denote the radius of the $n_{t h}$ decagram by $\mathrm{rad}_{n}$ and the next larger radius by $\mathrm{rad}_{n+1}$, then the ratio $\mathrm{rad}_{n+1} / \mathrm{rad}_{n}$ is equal to $\phi=(1+\sqrt{5}) / 2$. (Equation 1). This progression of nested decagrams serves a critical role in maintaining a relational aspect ratio between the different levels, which is the key to resolving the quasi-periodic structure.

$$
n_{0} \rightarrow n_{\infty}, \quad \frac{\operatorname{rad}_{n+1}}{\operatorname{rad}_{n}}=(1+\sqrt{5}) / 2
$$

The process for constructing the first-level hierarchy for Penrose pattern is demonstrated in Figure 3. Each cluster in the first hierarchy is composed of four building units; two 'seed' units with five-fold symmetry and their two fragments (Figure 3a). The sizes of the 'seed' units and their fragments are proportional to the size of the framework and is derived from the progression sequence of the nested decagrams (Figure 3c). The different combinations of the 'seed' units and their 'fragments' are shown in Figure $3 \mathrm{~b}$. The locations of the 'seed' units are determined by the intersection points generated by the framework (Figure 3d). The positions of the 'fragments' are guided by the position of the 'seed' units. The final arrangement of the first hierarchy of Penrose pattern is shown in Figure 3e. The construction of the global empire of the quasiperiodic formation requires building a progression of 


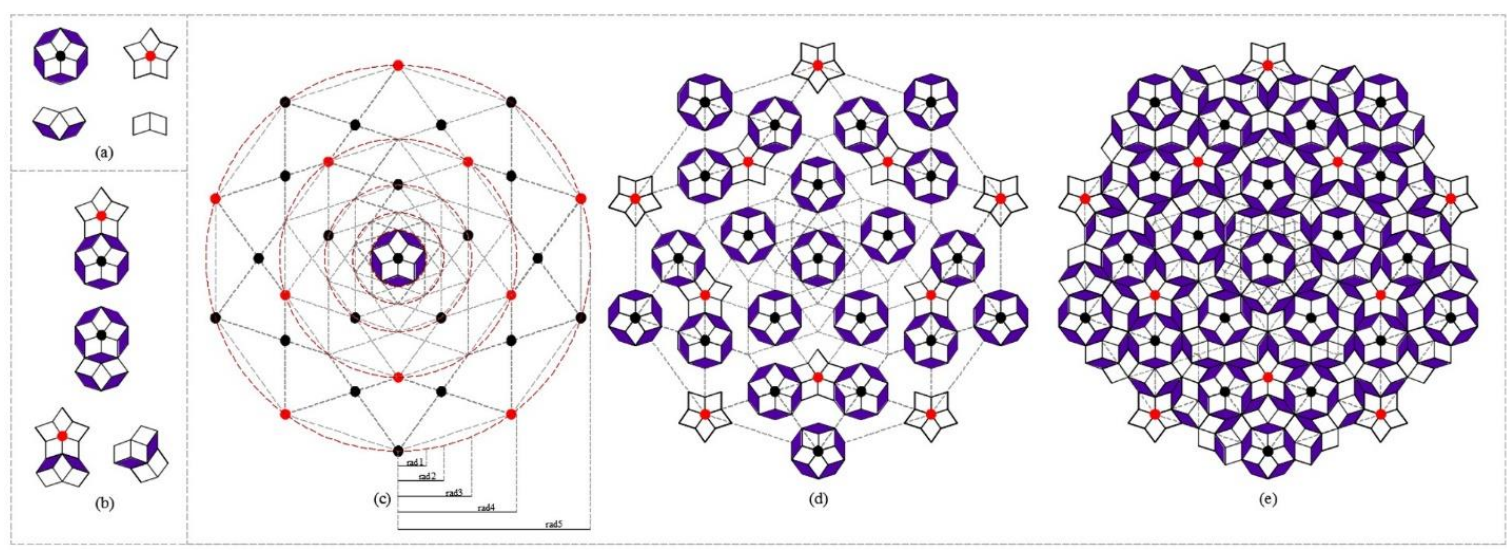

Figure 3 - A framework of nested decagrams based on $\phi$, serves as the underlying hidden grid for guiding the construction process of the quasi-periodic system.
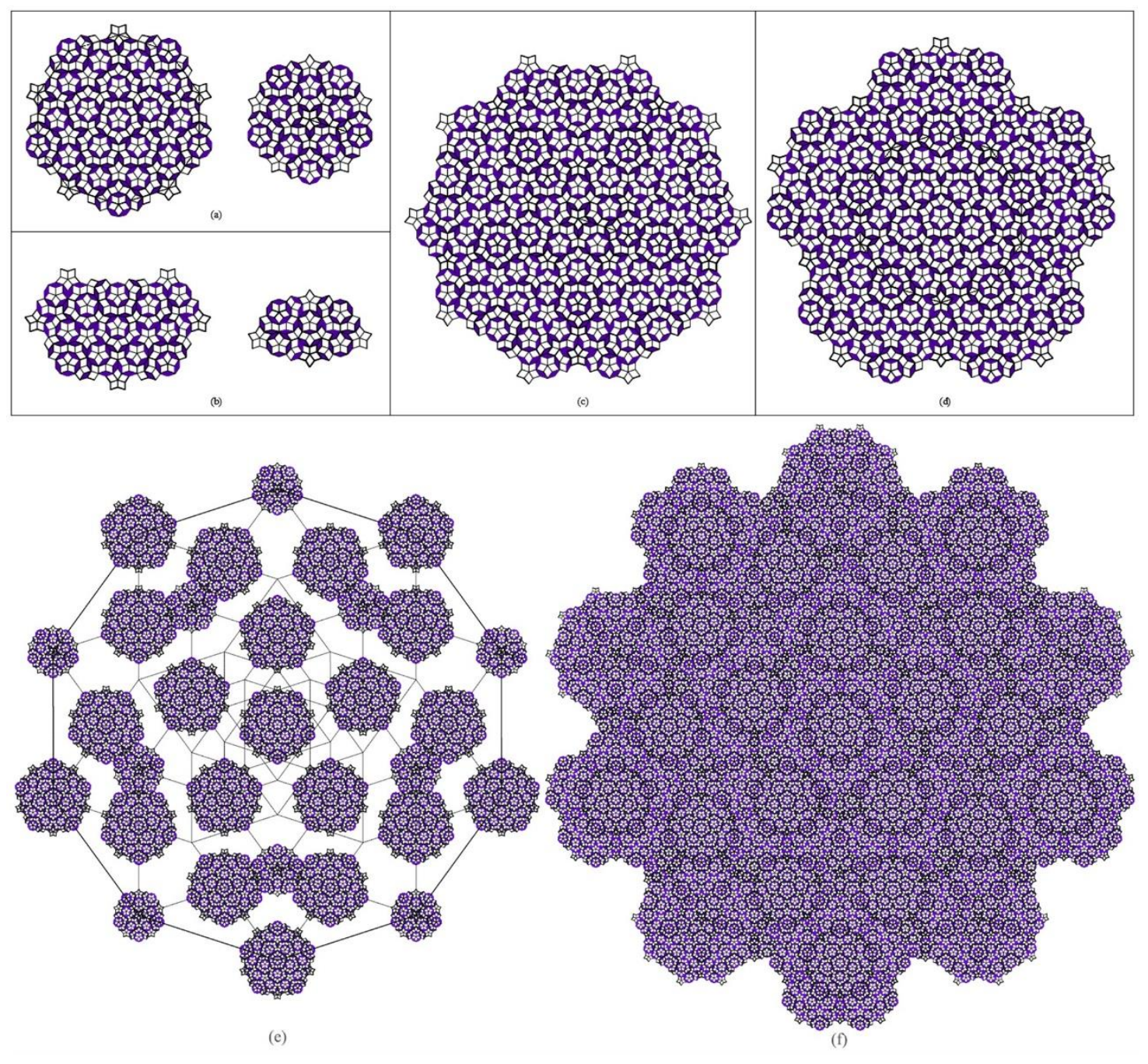

Figure 4 - The process for constructing the second-level hierarchy for Penrose tiling pattern. 


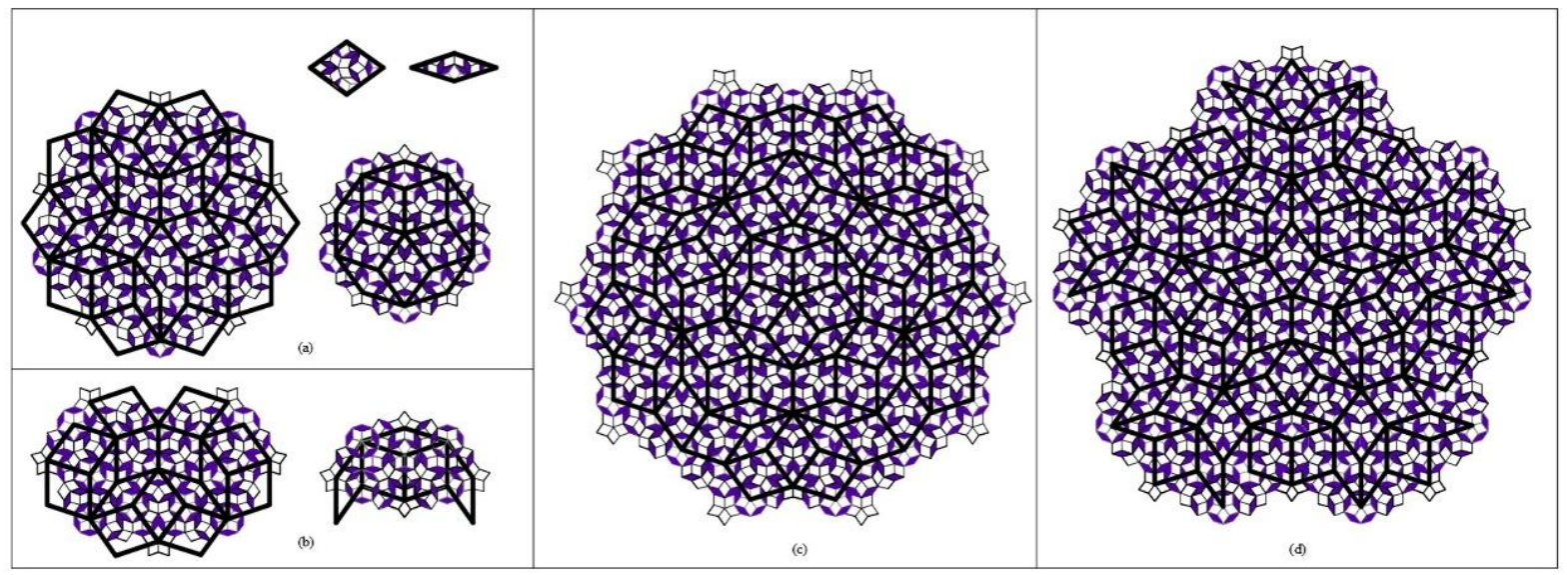

Figure $\mathbf{5}$ - Self-Similarity and deflation-inflation rules of Penrose quasi-periodic tiling pattern.
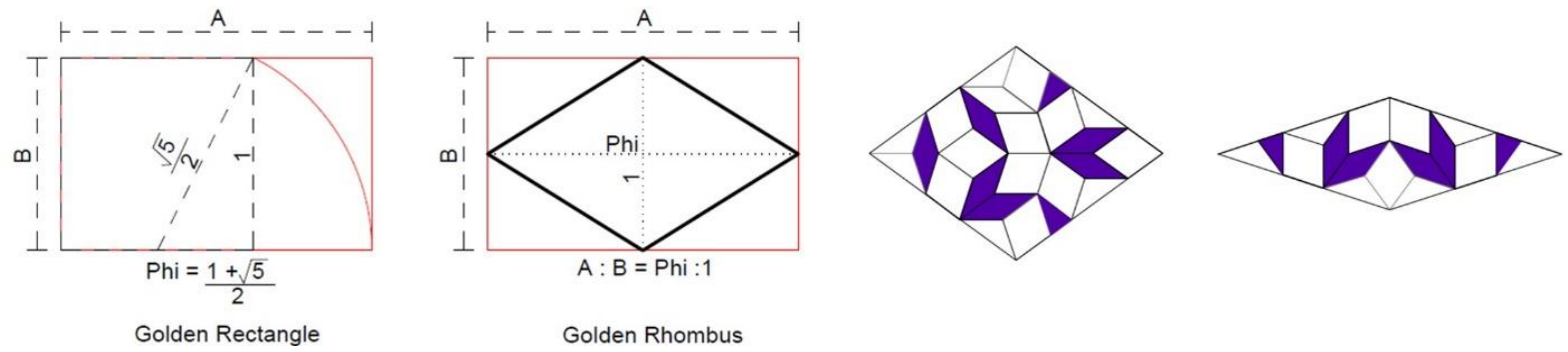

Figure 6 - Left: The golden rhombus. Right: the inflation/deflation rules of Penrose tiling.

order cluster is built on the lower order. In this system, the construction of the next higher-level is governed by a new generation of the nested decagrams, which grows proportionally according to the golden ratio and Fibonacci sequence.

The process for constructing the second-level hierarchy for Penrose pattern is demonstrated in Figure 4. In this second level, the final generated pattern of the first hierarchy serves as the seed cluster for the second hierarchy (Figure 4e). Each cluster in the second-level hierarchy is composed of four building clusters. The two 'seed' clusters (Figure $4 a)$ and their two fragments (Figure 4b), which are used to fill-in the gaps between the main 'seed' clusters. All units of the second-generation order are distributed according to the new generation framework of the nested decagrams (Figure 4e). The final arrangement of the second hierarchy of Penrose pattern is shown in Figure 4f. Accordingly to this sequence, generating the next higher-level cluster also follows the same process, in which the new higher-generation order is built on the previous order. An important characteristic of this multi-level proportional system is that the same elements of the patterns recur at different scales. This is often described as 'self-similarity' principle, which is the key principle in nature. A close-up detail of the generated Penrose pattern reveals the inflation rule of the second-hierarchy (Figure 5). According to these rules, the new generation thin and thick rhombus can be broken down to a smaller scale of the same pattern based on the golden ratio. In the next section, these deflation rules are used to generate the threedimensional surface profiles of Penrose patterns.

\subsection{2: Quasi-periodic surface design}

In order to construct the three-dimensional quasiperiodic (QP) surface design for Penrose tiling, it is important to understand that the two-dimensional Penrose formations are actually projections of the higher three-dimensional space. In this context, the thick and thin rhombus can be viewed as projections 

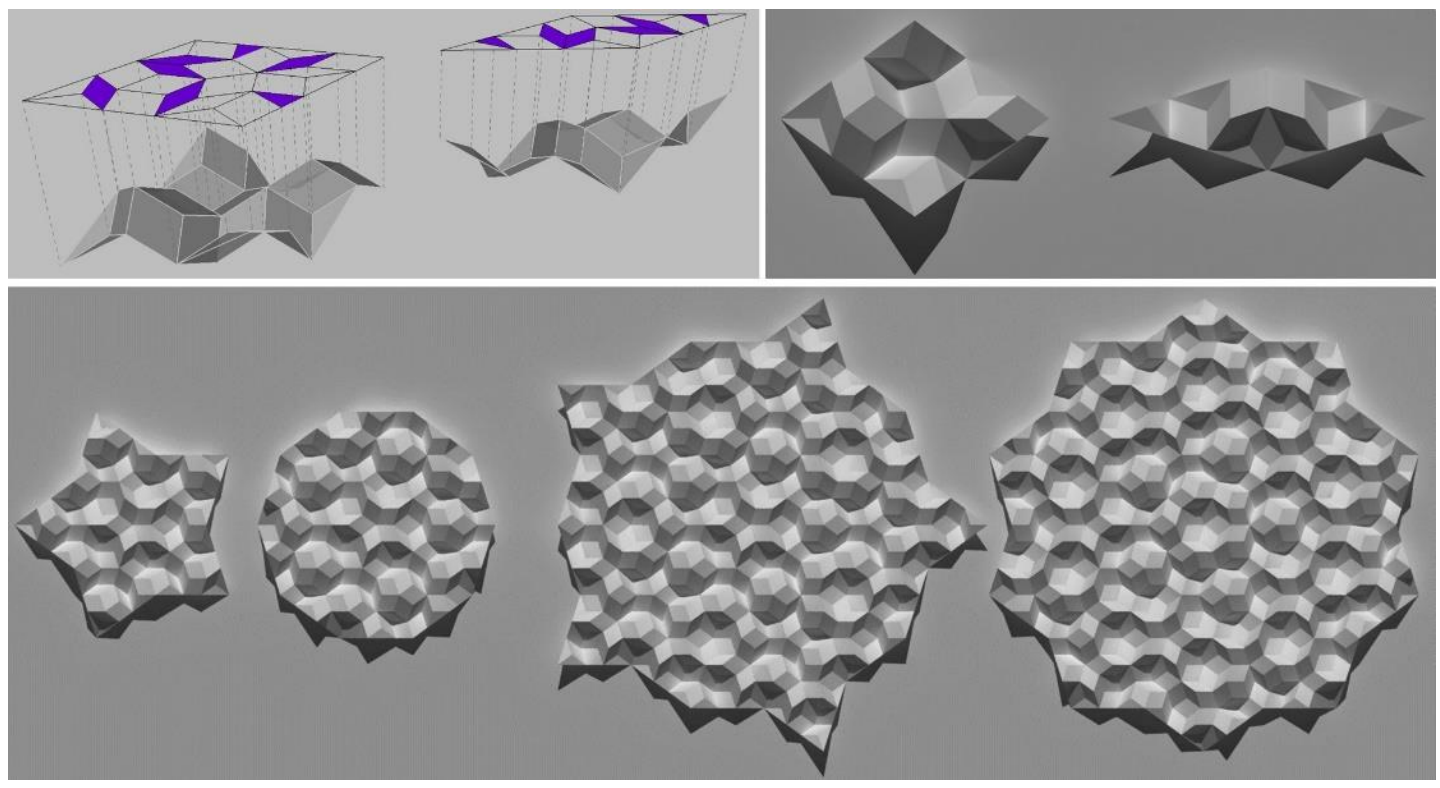

Figure 7 -Top: The two-dimensional Penrose tiling formations are projections of the golden rhombus in the threedimensional space. Bottom: The quasi-periodic surface design is generated by combining the second-level hierarchy of the thin and thick rhombus based on the deflation rules.

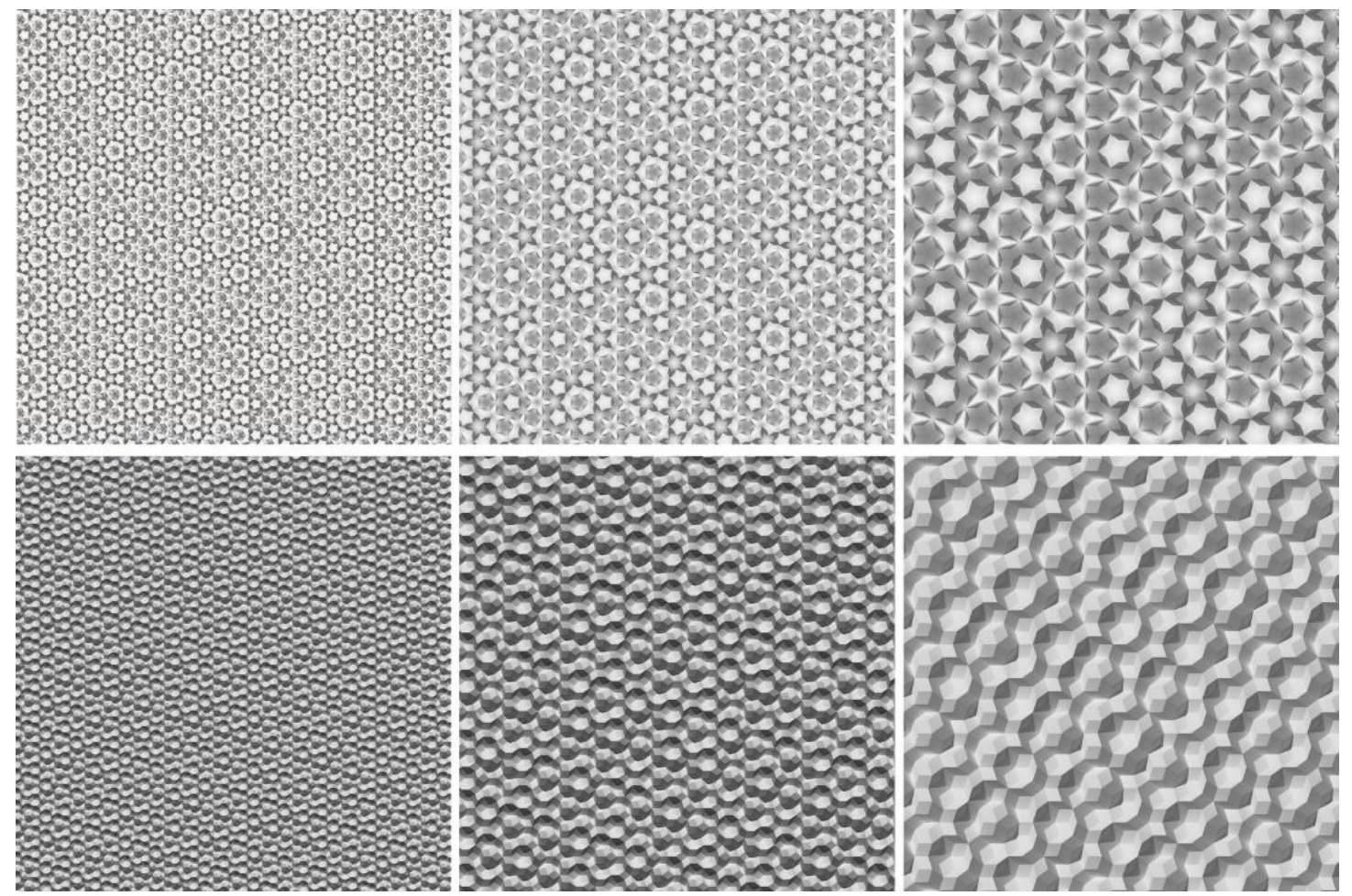

Figure 8-Rendered views of the quasi-periodic surface (top: vertical light, down: 45-degree light). 

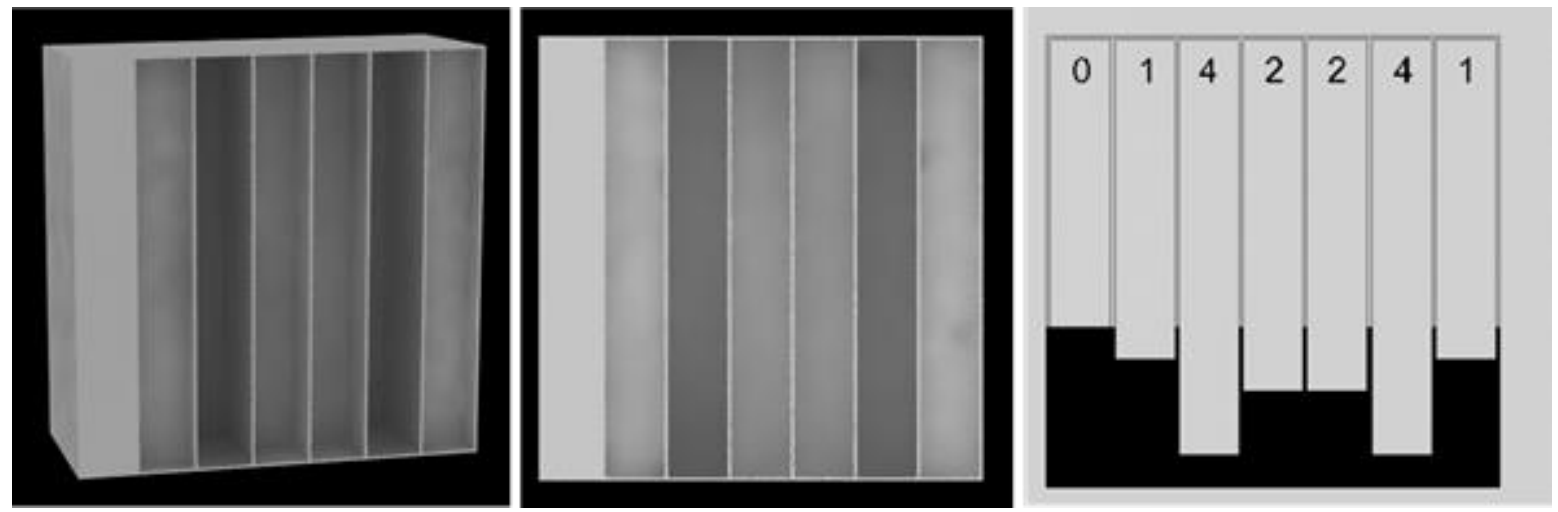

Figure 9-1D, N=7 Schroeder Quadratic Residue Diffuser.
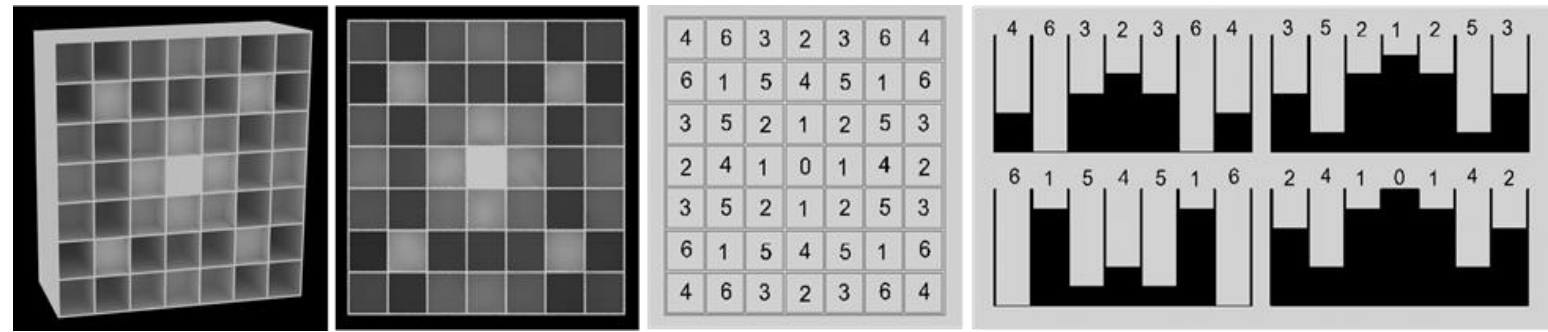

Figure $10-2 D, N=7 X 7$ Schroeder Quadratic Residue Diffuser.

two-dimensional plane. The golden rhombus is constructed by joining the mid points of the sides of a golden rectangle, in which the ratio of the long diagonal to the short diagonal equals to Phi (Figure 6). By reversing such projection, it is possible to reconstruct the three-dimensional surface structure of Penrose tiling using the deflation-inflation rules deduced in the previous section. The quasi-periodic surface design (Figure 7) is then generated by combining the second-level hierarchy of the thin and thick rhombus based on the deflation rules in Figure 5. Figure 8 shows different resolutions of the threedimensional quasi-periodic surface rendered in 90degree light (Figure 8-top) and 45-degree light (Figure 8-bottom). Interestingly, these rendered views also show visible traces of the Fibonacci bars rotated in the five directions. This demonstrates how simple rules can allow for the formations of complicated structures.

\subsection{3: 2D Quadratic Residue Diffuser (QRD)}

Figure 9 shows 1D Schroeder Quadratic Residue Diffuser; one of the most commonly used devices in the acoustics industry (Cox, and D'Antonio 2004).
This single plane diffuser consists of a series of seven wells with equal widths and different depths. The wells are separated by thin fins. The different depths are determined by the quadratic residue mathematical sequence, which is calculated based on the formula $\left(\right.$ depth $\left.=(\text { well position })^{\wedge} 2 \bmod N\right) . \mathrm{N}$ is a prime number and equals the number of wells. The N7 panel has depth sequence of $(0,1,4,2,2,4,1)$. The variations in depth causes sound waves to be reflected with phase delay based on the time it takes to travel down and up the well. To achieve the desired phase delay for a targeted wave, well depth should reach half of the wavelength to cause the phase change.

A single-plane 1D diffuser can cause diffuse reflection in a two-dimensional plane or into a hemi-disc. In order to generate diffuse reflections in threedimensional space or into a hemisphere, a multiplanes 2D diffuser is needed. Figure 10 shows a 2D QRD based on $N=7 X 7$. This specific array of sequences causes diffuse reflections along the vertical and horizontal planes as well as along the two diagonal directions (Cox, and D'Antonio 2004; Zhu et al. 2017). In this case, the diagonal sequence $(4,1,2,0,2,1,4)$ 

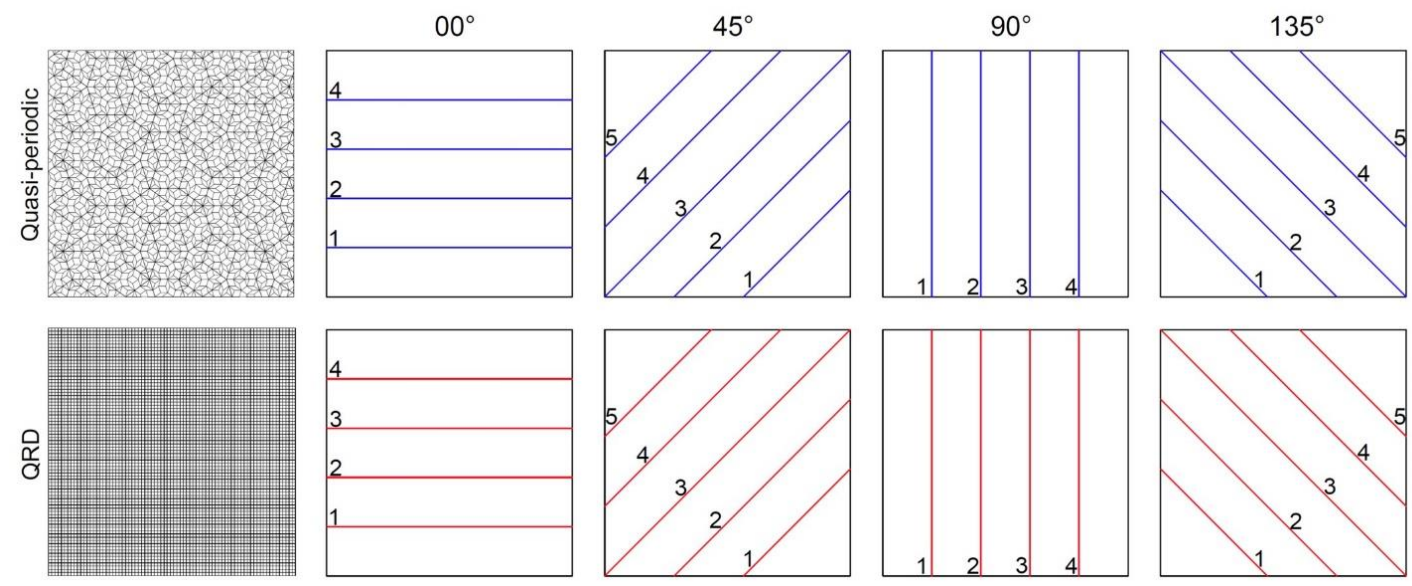

Figure 11 -For each of the two surfaces (Quasi-Periodic (QP) and the 2D Quadratic Residue Diffuser (QRD)) 18 different profiles were generated based on four directions.

\section{Quasi-Periodic}

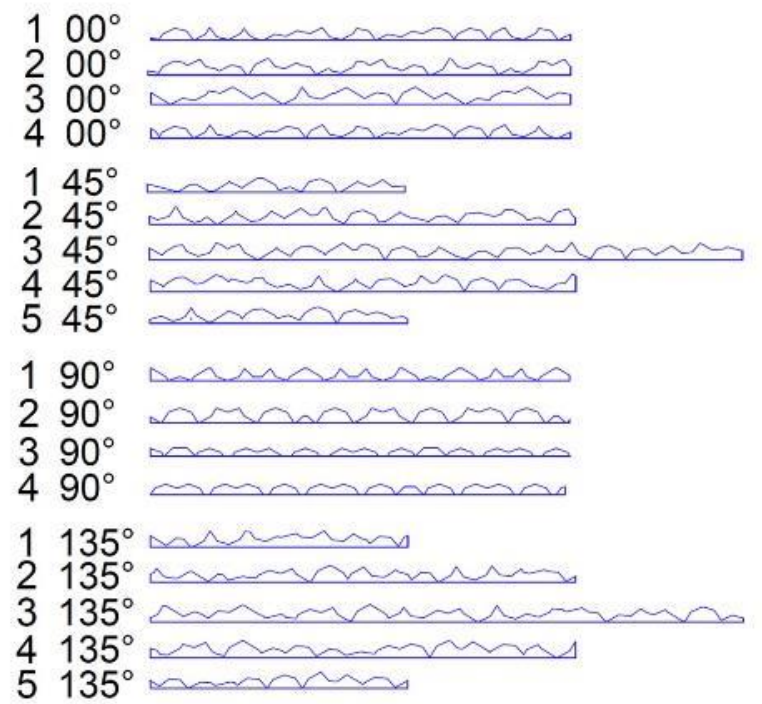

\section{QRD}

\begin{tabular}{|c|c|}
\hline $\begin{array}{l}00^{\circ} \\
00^{\circ} \\
00^{\circ} \\
00^{\circ}\end{array}$ & 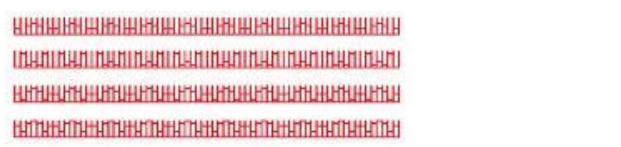 \\
\hline $\begin{array}{l}45^{\circ} \\
45^{\circ} \\
45^{\circ} \\
45^{\circ} \\
45^{\circ}\end{array}$ & 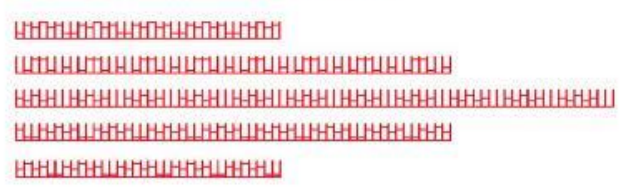 \\
\hline $\begin{array}{l}90^{\circ} \\
90^{\circ} \\
90^{\circ}\end{array}$ & 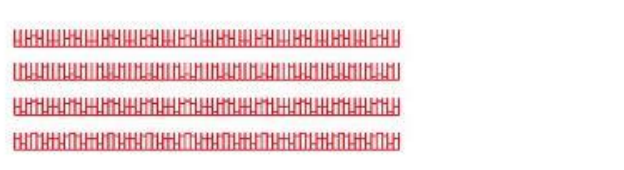 \\
\hline $\begin{array}{l}1 \\
1\end{array}$ & 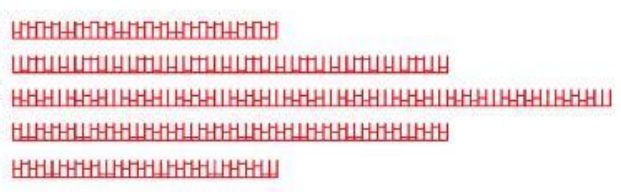 \\
\hline
\end{tabular}

Figure 12 -The generated sample profiles.

performs similarly to the original $1 \mathrm{D}$ sequence of $(0$, $1,4,2,2,4,1)$ and has the same Fourier properties. The four variations of surface cross-section profiles on the vertical and horizontal directions are shown in Figure 10 right.

In this research, the 2D 7X7 Schroeder type QRD provides a base-line performance measure for comparison. The extended 2D QRD diffuser is achieved by repeating the basic 7X7 unit with the assumption that it provides a true representative sample of the general periodic population. The depth sequence of the 2D QRD is designed to respond to a target frequency range between $900 \mathrm{~Hz}$ and $4000 \mathrm{~Hz}$. Accordingly, the max well depth is 5.86 inch, the well 
width is 1.85 inch and the fin width is 0.11 inch ${ }^{3}$. In this sequence the well number corresponds to the following depths in inches $\{0=0,1=.97,2=1.95,3=$ 2.93, $4=3.91,5=4.88,6=5.86\}$.

\subsection{4: Generating sample surface profiles}

For each of the two surface designs (Quasi-Periodic (QP) and the 2D Quadratic Residue Diffuser (QRD)) a square area of $12 \mathrm{ft} \times 12 \mathrm{ft}$ sample surface was constructed digitally (Figure 11). The profile depth of the QP diffuser was kept the same as the build depth of the QRD (5.86 inches). For each of the two surfaces 18 different profiles were generated based on four directions (horizontal, vertical and two diagonals) as shown in Figure 11. The profiles shown in figure 12 provide the sample data for testing the quality of the diffusers in terms of the spatial uniformity of the reflected sound energy.

\subsection{RESULTS AND DISCUSSION}

\subsection{The Normalized Diffusion Coefficients}

For each surface (QP and QRD), the normalized diffusion coefficients, based on diffuse field method was plotted for the 18 different sample profiles encompassing four different directions $(0,45,90$, and 135 degrees). The diffuse field method has the advantage of obtaining a quick random incident coefficient for predicting the performance of surface geometry. Figure 13 shows the normalized diffusion coefficient spectrums across the frequency range of $800 \mathrm{~Hz}$, to $4000 \mathrm{~Hz}$ for a total of 4 discrete profile samples for each surface (QP and QRD) in the horizontal direction (00 degrees). Figure 14 shows the normalized diffusion coefficient spectrums across the frequency range of $800 \mathrm{~Hz}$, to $4000 \mathrm{~Hz}$ for a total of 5 discrete profile samples for each surface (QP and $\mathrm{QRD}$ ) in the diagonal direction (45 degrees). Figure 15 shows the normalized diffusion coefficient spectrums across the frequency range of $800 \mathrm{~Hz}$, to $4000 \mathrm{~Hz}$ for a total of 4 discrete profile samples for each surface (QP and QRD) in the vertical direction (90 degrees). Figure 16 shows the normalized diffusion coefficient spectrums across the frequency range of $800 \mathrm{~Hz}$, to $4000 \mathrm{~Hz}$ for a total of 5 discrete profile samples for each surface (QP and QRD) in the other diagonal direction (135 degrees). The results clearly show that, in all tested directions, the normalized diffusion coefficients values for the QP surface (Blue line) performed better than QRD surface (Red line).

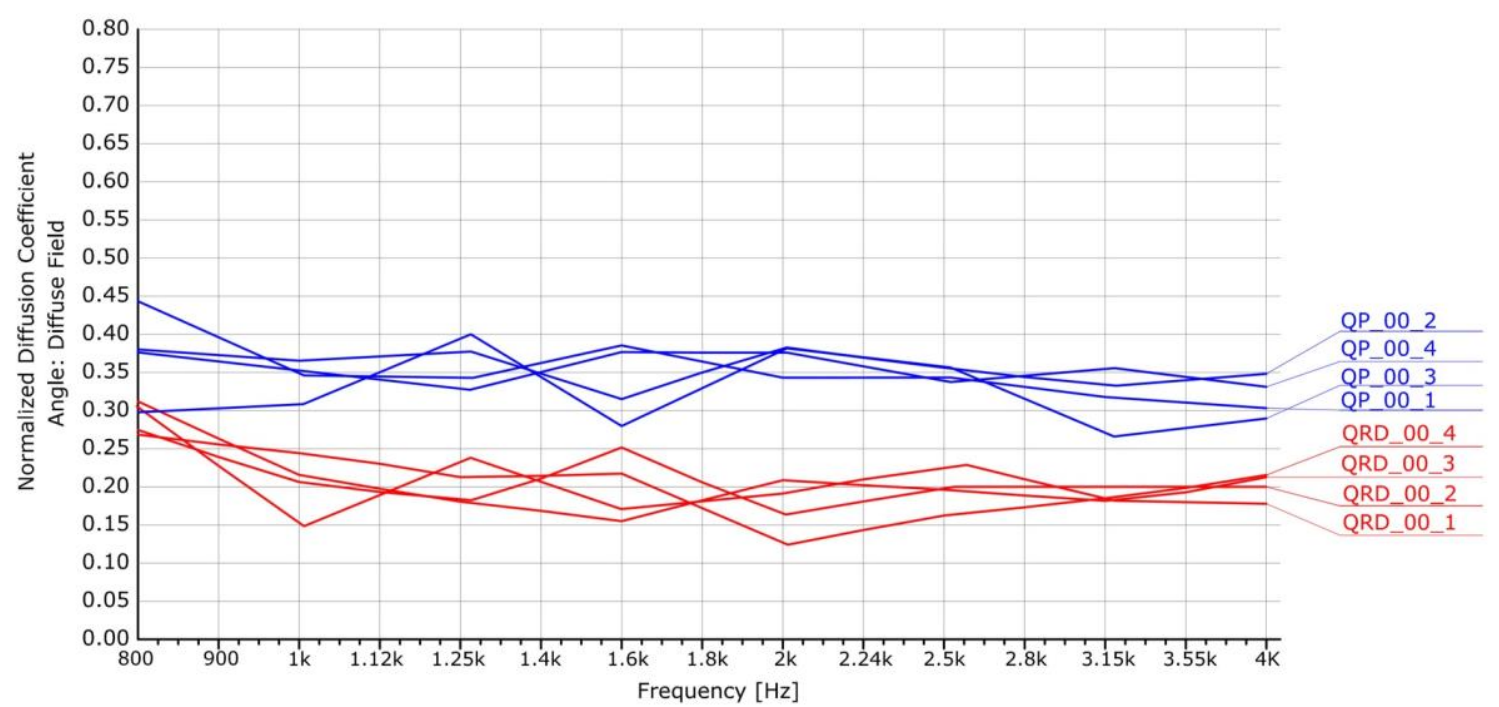

Figure 13 -The normalized diffusion coefficient spectrums across the frequency range of $800 \mathrm{~Hz}$, to $4000 \mathrm{~Hz}$ for a total of 4 discrete profile samples for each surface (QP and QRD) in the horizontal direction (00 degrees).

3 All measurements are calculated using QRDude

V:3.10:https://www.subwoofer-

builder.com/qrdude.htm 


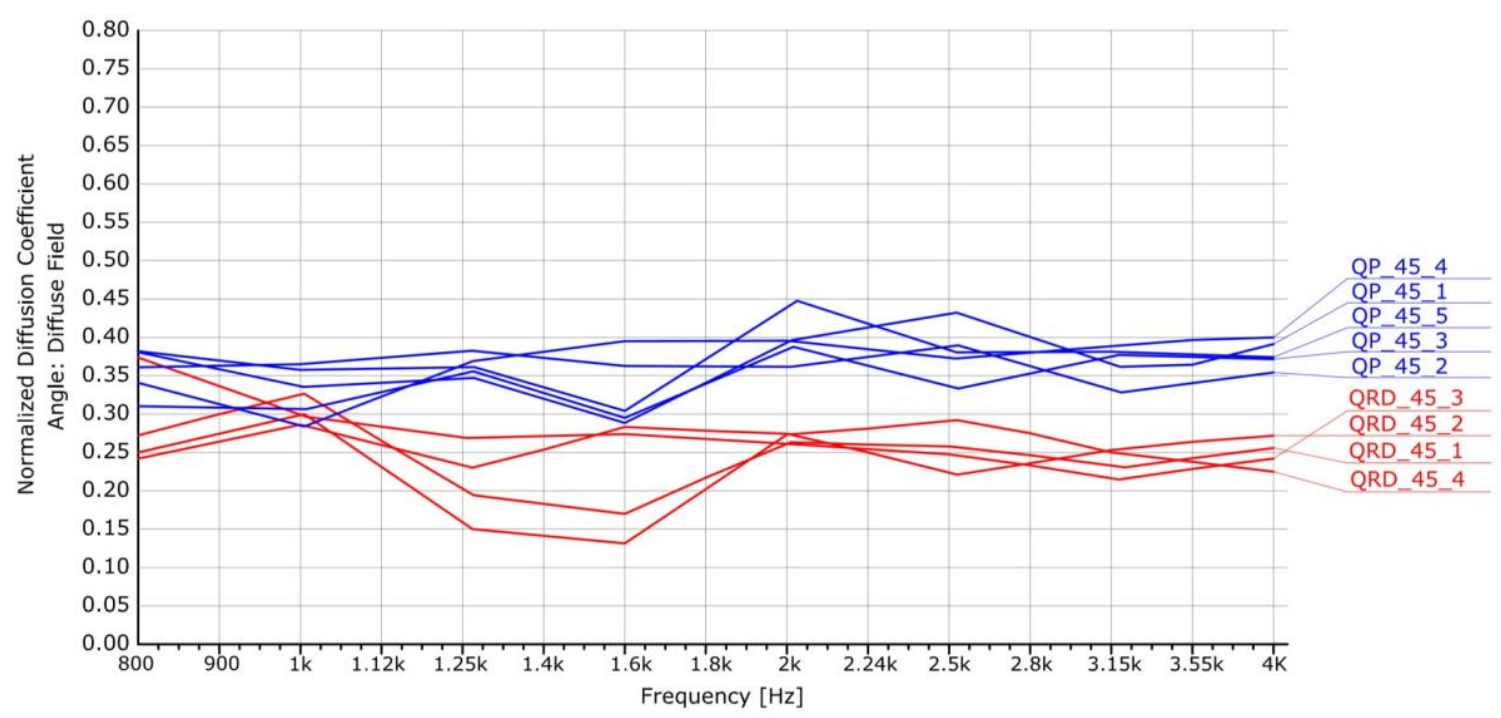

Figure 14 - The normalized diffusion coefficient spectrums across the frequency range of $800 \mathrm{~Hz}$, to $4000 \mathrm{~Hz}$ for a total of 5 discrete profile samples for each surface (QP and QRD) in the diagonal direction (45 degrees).

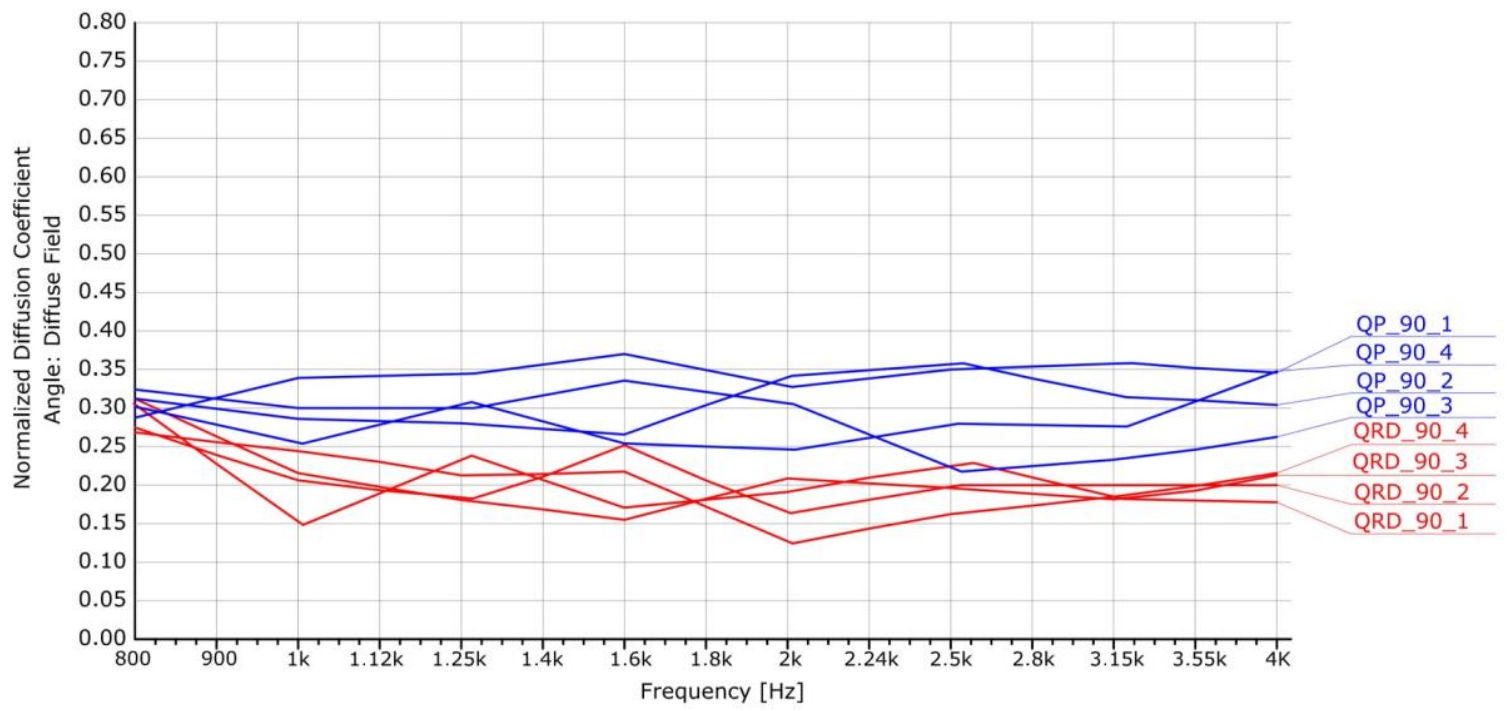

Figure 15 - The normalized diffusion coefficient spectrums across the frequency range of $800 \mathrm{~Hz}$, to $4000 \mathrm{~Hz}$ for a total of 4 discrete profile samples for each surface (QP and QRD) in the vertical direction (90 degrees). 


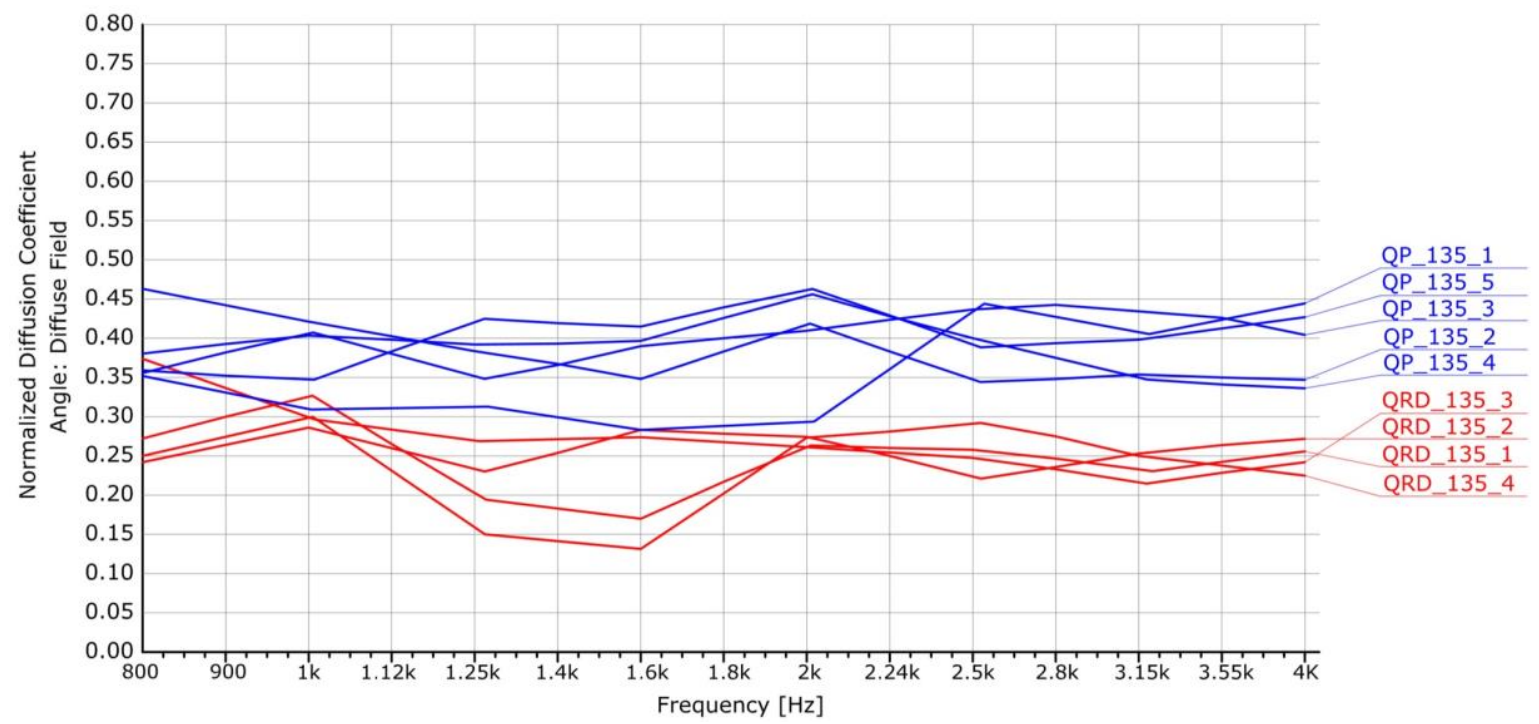

Figure 16 - The normalized diffusion coefficient spectrums across the frequency range of $800 \mathrm{~Hz}$, to $4000 \mathrm{~Hz}$ for a total of 5 discrete profile samples for each surface (QP and QRD) in the other diagonal direction (135 degrees).

The uniformity of the sound energy reflected from surfaces can be measured as a spatial polar response in one-third octaves for a specific angle of incidence, which is used to characterize the diffuser's performance. For each of the 18 sample profiles, the Spatial Response Plot of the reflected sound wave was calculated for three different angles of incidence $(-45,0$ and 45$)$ and for 4 different frequencies (1000 $\mathrm{Hz}, 2000 \mathrm{~Hz}, 3150 \mathrm{~Hz}$ and $4000 \mathrm{~Hz}$ ). Figure 17 shows the spatial response plot for a total of 4 discrete sample profiles for each surface (OP and QRD) in the horizontal direction ( 00 degrees). Figure 18 shows the spatial response plot for a total of 5 discrete sample

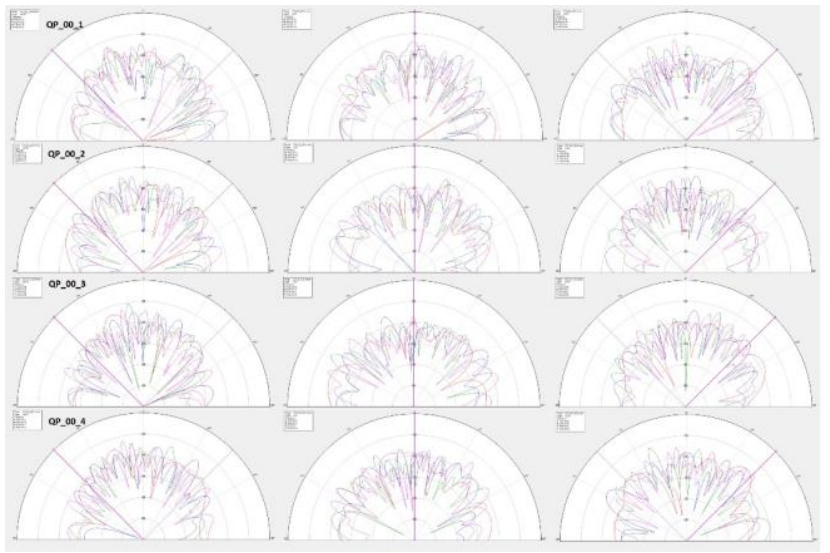

profiles for each surface (OP and QRD) in the diagonal direction (45 degrees). Figure 19 shows the spatial response plot for a total of 4 discrete sample profiles for each surface (OP and QRD) in the vertical direction (90 degrees). Figure 20 shows the spatial response plot for a total of 5 discrete sample profiles for each surface (OP and QRD) in the other diagonal direction (135 degrees). The results show better uniform distributions associated with the QP surface. Moreover, the scattered polar responses for the QRD surface show grating lobes generated by the fact that it is periodic. Such lobes increase with the number of repetitive units in the surface design.

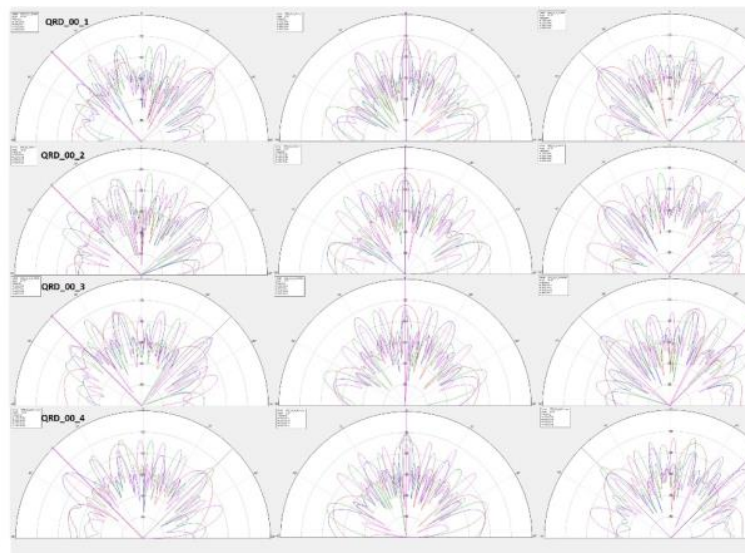

Figure 17 - The spatial response plot for a total of 4 discrete sample profiles for each surface (OP and QRD) in the horizontal direction (00 degrees). 


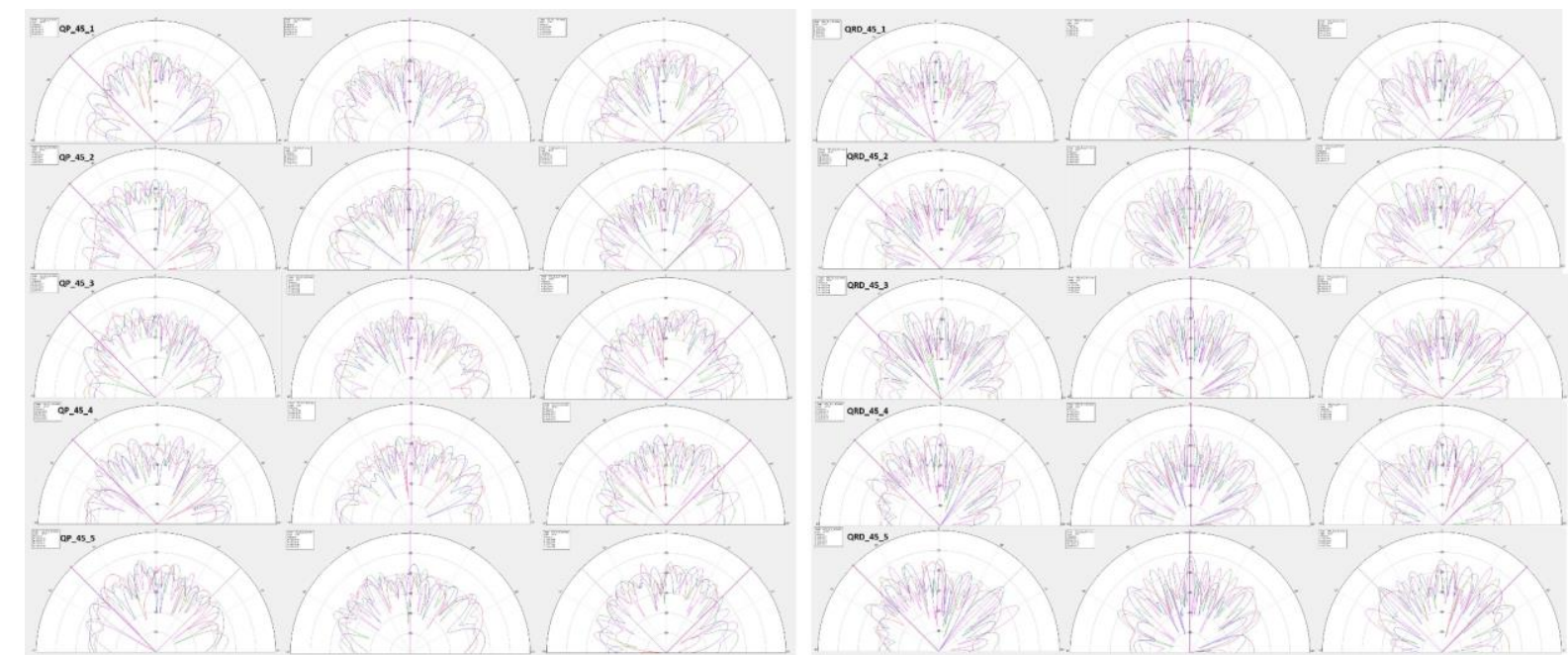

Figure 18 -The spatial response plot for a total of 5 discrete sample profiles for each surface (OP and QRD) in the diagonal direction (45 degrees).
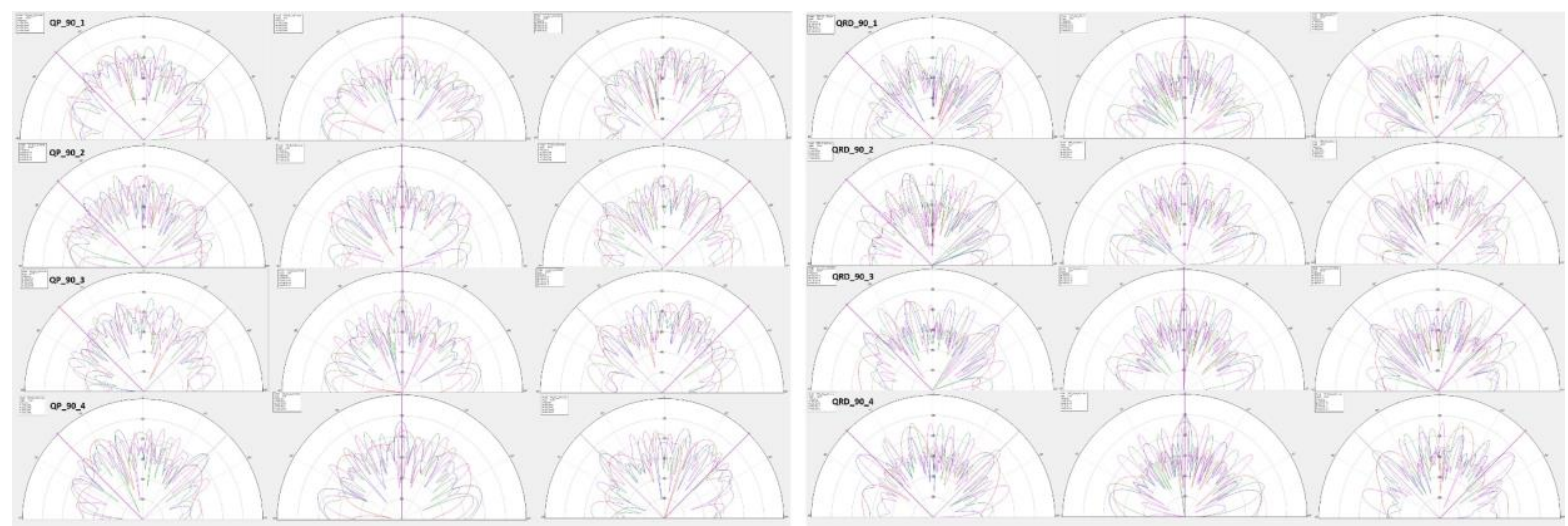

Figure 19 -The spatial response plot for a total of 4 discrete sample profiles for each surface (OP and QRD) in the vertical direction (90 degrees).
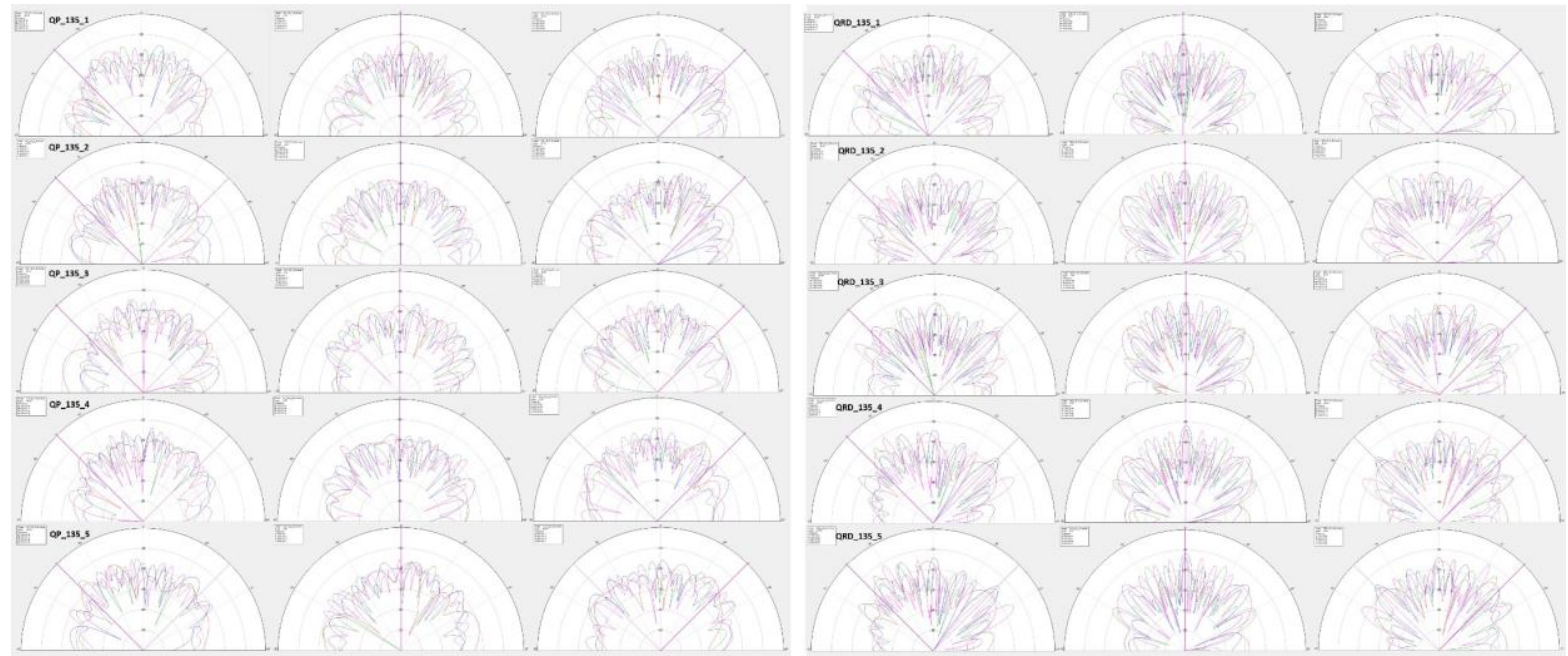

Figure 20 -The spatial response plot for a total of 5 discrete sample profiles for each surface (OP and QRD) in the other diagonal direction (135 degrees). 


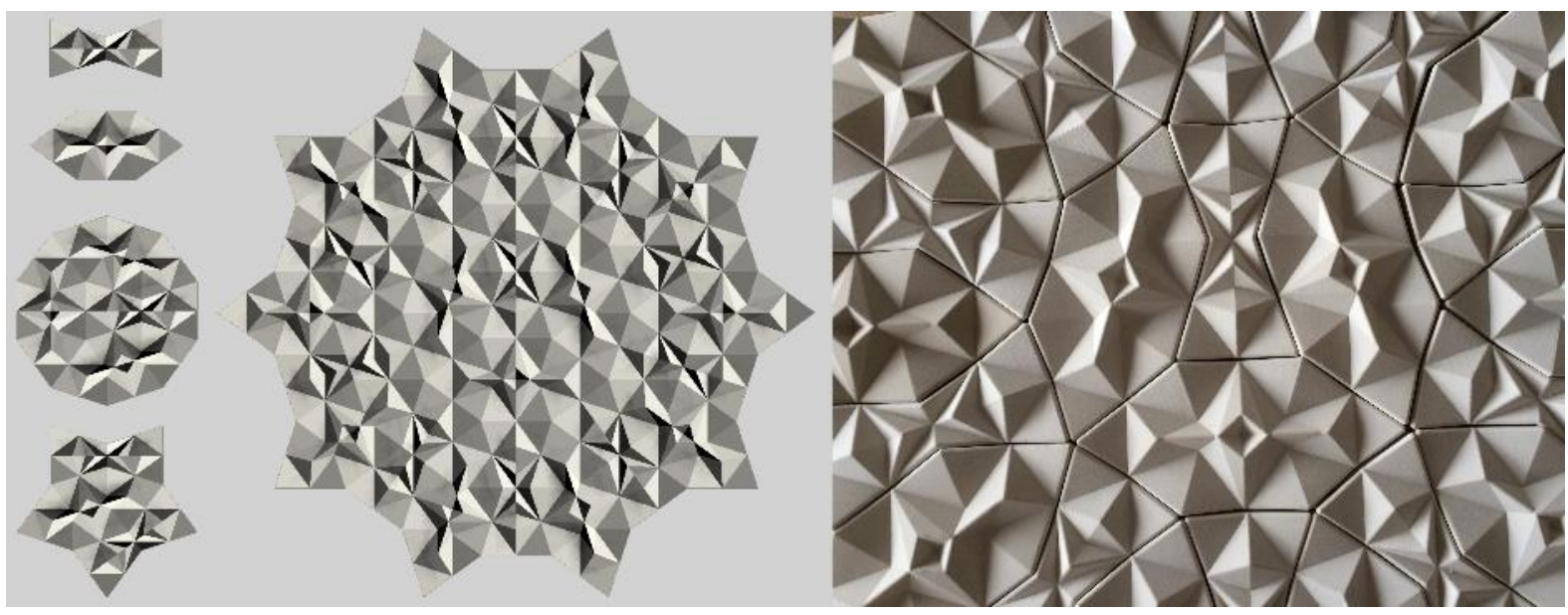

Figure 21 - 10-fold Quasi-periodic surface panel fabricated with porcelain slip casting method using two modular units.

\subsection{New Surface Designs for Architecture Acoustics.}

In this paper, a new approach for acoustical surface design based on quasi-periodic formations is presented. While this study focuses specifically on Penrose-based surfaces, the range of design possibilities is wide and allows for variations while preserving the quasi-periodic symmetry. Moreover, these designs are built on hierarchical structures that allow self-similar systems to emerge, which can be used to optimize surface designs for architectural acoustics. Because of the limited number of the basic building units, these surfaces can be easily fabricated using simple casting processes. The example in Figure 21 shows a 10 -fold quasi-periodic surface design consisting of two basic units. This tiling system can be easily fabricated using ceramic slip casting methods (figure 21 right).

Figure 22 demonstrates the process for developing the surface design in Figure 21 . The construction process utilizes a simple relational logic that can be used to generate variety of non-periodic hierarchical designs (Ajlouni2018). In general, generating the quasi-periodic formations is based on utilizing two design components, an underlying structural grid and a (seed) units. In this formula, the underlying grid provides the relational logic for mapping the locations of the repeated seed units and is responsible for defining the underlying symmetry. By manipulating the design of the seed units, a wide range of variations can be achieved. Generating the structural grid is attained by building a progression of nested decagrams, in which, every hierarchy is proportionally built on the previous one; resulting in a self-similar network (Figure 22 a). The intersection points on the network define the locations of the seed units (Figure $22 \mathrm{~b}$ ). In this system, the connecting formations between the main seed units are flexible and will not affect the underlying symmetry (Figure $22 \mathrm{c}$ ). By analyzing the generated design in figure 22 $\mathrm{d}$, it is possible to define two basic modular units. The profile and depth of these two units can be customized to respond to specific acoustic design challenge.

Quasi-periodic surface profiles can be applied in many design situations to improve the audible performance. For example, these designs can be used on rear walls of large auditoria to prevent echoes (Cox and D'Antonio 2004). Such strategy preserves the energy from the sound field, which presents an advantage over using absorbers. Similar diffusers can also be used in small rooms with parallel walls to eliminate flutter echoes. Small rooms can also benefit from similar diffusers, which can promote a passive background sound while minimizing the coloration effects of early reflections (Cox et al. 2006). Moreover, these designs can be used to reduce effects of early arriving reflections especially in large spaces with low ceilings (Cox and D'Antonio 2004) as well as to improve sound performance of long and narrow spaces (i.e. subway stations).

By utilizing the qualities of quasi-periodic structures, this research hopes to inspire a new wave of acoustical surface designs that allows the designer to 


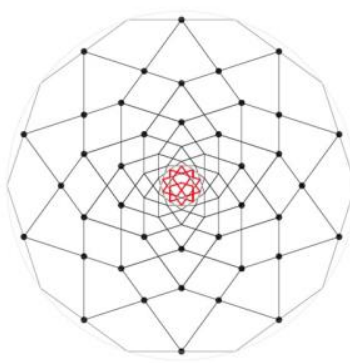

(a)

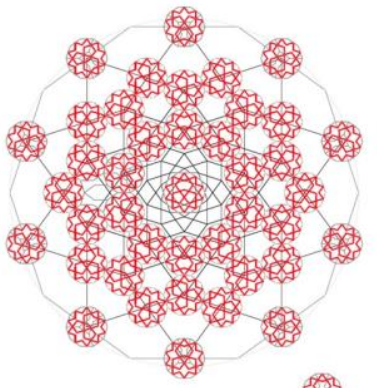

(b)

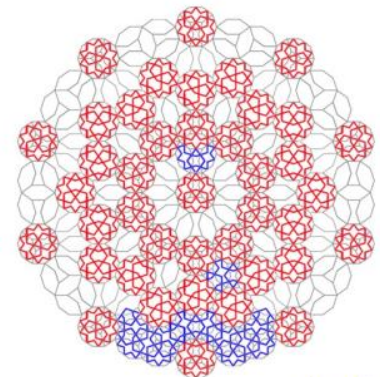

(c)

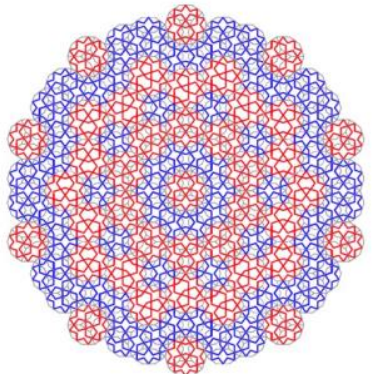

(d)
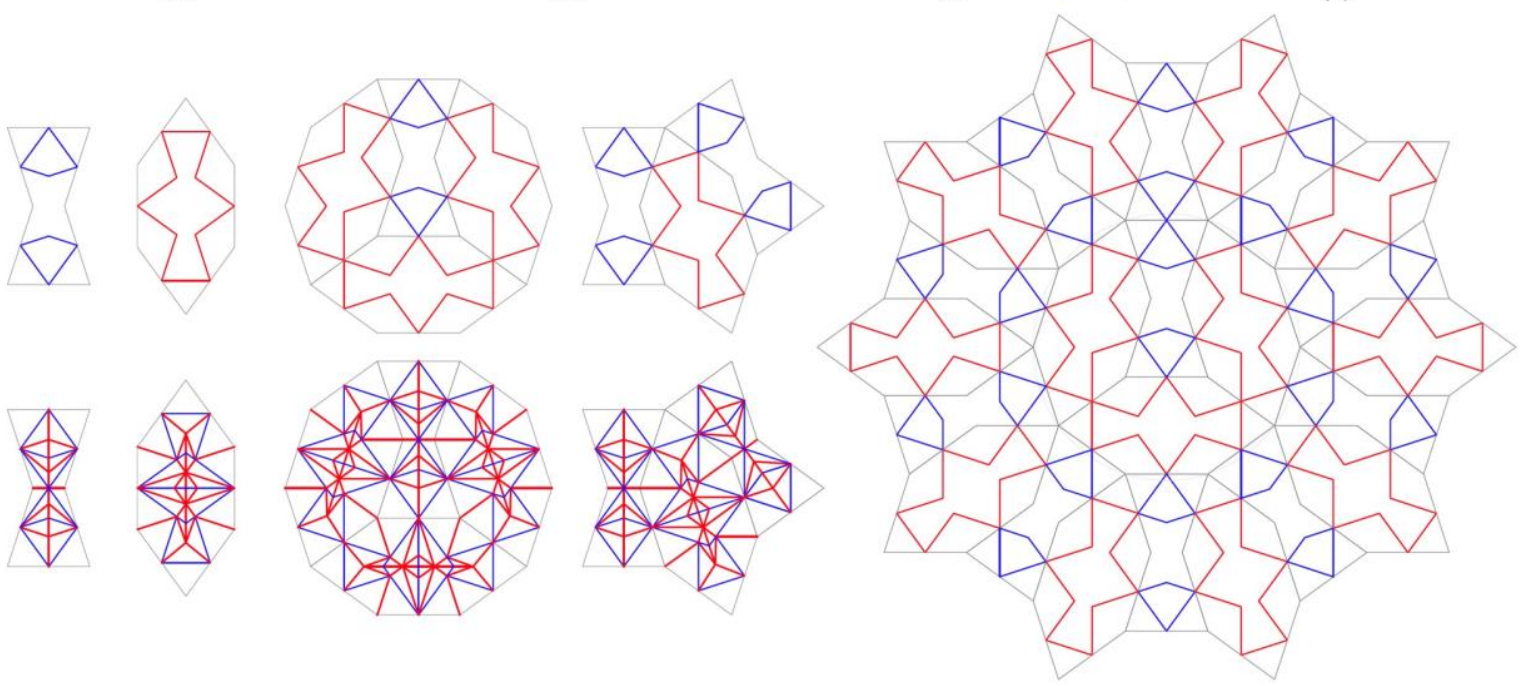

Figure 22 - The process for developing the surface design in Figure 21.

encode a wide range of behavioral and aesthetic qualities.

\subsection{CONCLUSION}

This research is set to explore the potential of using quasi-periodic formations for designing surface diffusers. A new type of quasi-periodic surface diffuser based on 2D Penrose tiling formations is developed. The quality of the diffuse reflection caused by the quasi-periodic surface was compared to the diffuse reflection caused by Schroeder's 2D Quadratic Residue Diffuser (QRD). The numerical results show that the Quasi-periodic diffuser performed better than the 2D (QRD). The quasiperiodic surface designs have two main performative qualities, which make them more appealing than periodic surface designs. The first advantage relates to the fact that quasi-periodic designs do not repeat their formations while expanding to fill the surface. This quality allows for every quasi-periodic profile to have a distinct design, which in turn produces a completely distinct sound reflection pattern. This is an essential property for an effective scattering performance, by which, eliminating the formations of orchestrated reflections and thus allowing for a wider range of distribution of the reflection rays. Periodic designs, on the other hand, repeat the same formations based on the number of basic units, which often produce the same profiles resulting in the reoccurrence of the exact reflection patterns. This can amplify certain directions; producing patterns of sound reflections (i.e. comb filtering). The second advantage of utilizing the quasi-periodic structures for designing sound diffusers, is their encoded selfsimilar properties, which allow the same nonrepeating formations to appear at multiple scales. This quality has the potential for optimization to respond to a wider band of sound wavelengths. 
Moreover, the introduced process for developing quasi-periodic surface designs is flexible and provides a wider space for creativity and design aesthetics.

While these results provide preliminary indicators for the diffusion behavior of the sample data, follow-up investigations should include the use of physical experimentation to validate the numerical results. Future research should also investigate the diffusion properties of other non-periodic formations, including 7-fold, 8-fold, 9-fold, 10-fold, 12-fold, etc., as well as designing and testing optimized hybrid designs than encode the qualities of Schroeder's profiles within the quasi-periodic arrangements.

\section{Funding information}

This research was funded, in part, by the 2015 University of Utah Faculty Research \& Creative Grant Projects

\section{References}

Abe, Eiji, Yanfa Yan and Stephen J. Pennycook. 2004. Quasicrystals as cluster aggregates. Nat. Mater. 3:759. https://doi.org/10.1038/nmat1244

AFMG Technologies GmbH, 2011-2018. AFMG Reflex - A new tool for the design and application of diffuser. Ahnert Feistel Media Group. Berlin, Germany. Accessed online at http://reflex.afmg.eu/) on July 102018.

Ajlouni, R. 2011. “A long-range hierarchical clustering model for constructing perfect quasi-crystalline formations". Philosophical Magazine 91:2728-2738 https://doi.org/10.1080/14786435.2010.532515

Ajlouni, R. 2012. "The global long-range order of quasiperiodic patterns in Islamic architecture". Acta Crystallographica Section A. 68: 235-243. https://doi.org/10.1107/S010876731104774X

Ajlouni, R. 2013. "Octagon-based quasi-crystalline formations in Islamic architecture". In Aperiodic Crystals, ed. Siegbert Schmid, Ray L. Withers and Ron Lifshitz, 4958. Amsterdam: Springer.

Ajlouni, R. 2017a. "Simulation of sound diffusion patterns of fractal-based surface profiles." In ACADIA 2017: DISCIPLINES \& DISRUPTION, Proceedings of the 37th Annual Conference of the Association for Computer Aided Design in Architecture (ACADIA) ISBN 978-0-692-96506-1. Cambridge, MA 2-4 November, 2017, pp. 52-61.
Ajlouni, R. 2017b. "An ancient rule for constructing dodecagonal quasi-periodic formations". Journal of Physics: Conference Series. Volume 809 (1): http://iopscience.iop.org/article/10.1088/17426596/809/1/012028/pdf

Ajlouni, R. 2018. "The relational logic behind the hierarchical geometry in Islamic art." In Proceedings of ISSC 2018: Logics of Image: Visual Learning, Logic and Philosophy of Form in East and West, Kolymbari, Crete, 1118.

Angus, J. A. 1995. "Using modulation phase reflection grating to achieve specific diffusion characteristics." In the 99 Audio Engineering Society Convention, pre-print 4117.

Arau-Puchades, H. 2016. "Sound is a wave: A new concept of Huygens acoustics diffuser" In Concert Hall Acoustics. PROCEEDINGS of the 22nd International Congress on Acoustics, Paper ICA2016-32. Buenos Aires -5 to 9 September, 2016.

Bak, P. 1986. Icosahedral crystals: Where are the atoms?. Phys. Rev. Lett. 56: 861-864.

https://doi.org/10.1103/PhysRevLett.56.861

Boriskina, SV. 2015. Quasicrystals: Making invisible materials. Nat Photonics 9:422-424.

https://doi.org/10.1038/nphoton.2015.107

Bradley, David, Erik O. Snow, Kimberly A. Riegel, Zachary D. Nasipak, and Andrew S. Terenzi. 2011. "Numerical prediction of sound scattering from surfaces with fractal geometry: A preliminary investigation." Proc. Mtgs. Acoust. 12: 015010.

Cox, T. J. 1996. "Designing curved diffusers for performance spaces." J. Audio. Eng. Soc. 44: 354-364.

Cox, T. J. and P. D'Antonio. 1997. "Fractal sound diffusers." Proc. of the 103rd Convention of the Audio Eng. Soc. 1- 12.

Cox, T. J. and Y. Lam. 1993. "Evaluation of methods for predicting the scattering from simple rigid panels." Applied Acoustics. 40: 123-140. https://doi.org/10.1016/0003682X(93)90086-L

Cox, T. J. and Y. Lam. 1994. "Prediction and evaluation of the scattering from quadratic residue diffusers." J. Acoust. Soc. Am. 95: 297-305. https://doi.org/10.1121/1.408361 
Cox, Trevor J. and P. D'Antonio. 2004. Acoustic Absorbers and Diffusers: Theory, Design and Application. 1st ed. London and New York: Taylor \& Francis.

Cox, Trevor J. and P. D'Antonio. 2009. Acoustic Absorbers and Diffusers: Theory, Design and Application. 2nd ed. London and New York: Taylor \& Francis.

Cox, Trevor J. and P. D'Antonio. 2017. Acoustic Absorbers and Diffusers: Theory, Design and Application. 3rd ed. Boca Raton, FL: CRC Press.

Cox, T. J., B. I. L. Dalenback, P. D'Antonio, J. J. Embrechts, J.Y.Jeon, E. Mommertz and M. Vorl'aner. 2006." A tutorial on scattering and diffusion coefficients for room acoustic surfaces." Acta Acustica united with Acustica 92(1):1-15.

D'Antonio, P. and J. H. Konnert. 1992. "The QRD diffractal: A new one- or two-dimensional fractal sound diffusor." J. Audio Eng. Soc. 40: 117-129.

D'Antonio, P. and T. J. Cox. 2000. "Diffusor application in rooms." Applied Acoustics 60:113-42.

https://doi.org/10.1016/S0003-682X(99)00054-7

D'Antonio, P. and T. J. Cox. 2004. Embodiments of aperiodic tiling of a single asymmetric diffusive base shape. US patent 6772859.

De Bruijn, N. G. 1981a. "Algebraic theory of Penrose's nonperiodic tilings of the plane I." Indag. Math. 43:39-52. https://doi.org/10.1016/1385-7258(81)90016-0

De Bruijn, N. G. 1981b. "Algebraic theory of Penrose's nonperiodic tilings of the Plane II." Indag. Math. 43:53-66. https://doi.org/10.1016/1385-7258(81)90017-2

De Bruijn, N. G. 1981c. "Sequences of zeros and ones generated by special production Rules." Indag. Math. 43:27-37. https://doi.org/10.1016/1385-7258(81)90015-9

Dong J.W., M.L. Chang, X.Q. Huang, Z.H. Hang, Z.C. Zhong, W.J. Chen and S.V. Boriskina. 2015. "Conical dispersion and effective zero refractive index in photonic quasicrystals." Phys. Rev. Lett. 114(16): 163901. https://doi.org/10.1103/PhysRevLett.114.163901

Dubois, Jean-Marie. 2012. "Properties- and applications of quasicrystals and complex metallic alloys." Chem. Soc. Rev. 41: 6760-6777. https://doi.org/10.1039/c2cs35110b

Embrechts, J., D. Archambeau, and G. B. Stan. 2001. "Determination of the scattering coefficient of random rough diffusing surfaces for room acoustics applications." Acta Acustica united with Acustica 87: 482-494.

Everest, F. and K. Pohlmann. 2009. Master Handbook of Acoustics. 5th ed. New York: McGraw Hill.

Farner, J. 2014. Acoustic diffusion: Simulation and Investigation of 2D Diffusers using the Boundary Element Method. BE (Hons) thesis, University of Tasmania: Hobart.

Fay, Michael W. 2013. "Acoustics 101 for architects." The Journal of the Acoustical Society of America 134: 4005. https://doi.org/10.1121/1.4830611

Fujiwara K. 1995. "A study on the sound absorption of a quadratic-residue type diffuser." Acta Acust United Acust 81:370-8.

Funkhouser, T.,Tsingos T., Carlbom I., Elko G., Sondhi, M. and West, J. 2002. "Modeling sound reflection and diffraction in architectural environments with beam tracing." Forum Acusticum, THE 3rd EAA EUROPEAN CONGRESS ON ACOUSTICS. Seville, Spain: 8.

Hargreaves, T., Cox, T. and Lam, Y. 2000. "Surface diffusion coefficients for room acoustics: Free-field measures." J.

Acoust. Soc. Am. 108: 1710-1720.

https://doi.org/10.1121/1.1310192

Henham, W, D. Holloway and L. Panton. 2016. "Broadband acoustic scattering with modern aesthetics from random 3D terrain surfaces generated using the Fourier Synthesis algorithm." In Proceedings of Acoustics 2016: The Second Australasian Acoustical Societies Conference, Brisbane, Australia: 1-10.

Hughes, R.J., J.A. Angus, T.J. Cox, O. Umnova, G.A. Gehring, M. Pogson, and D. M. Whittaker. 2010.

"Volumetric diffusers: pseudorandom cylinder arrays on a periodic lattice." J Acoust Soc Am.128(5):2847-56. https://doi.org/10.1121/1.3493455

Ishii, Y. and T. Fujiwara. 2008. "Electronic Structures and Stability of Quasicrystals." In Quasicrystals, edited by T. Fujiwara and Y. Ishii, 171- 203. Amsterdam: Elsevier. https://doi.org/10.1016/S1570-002X(08)80021-3

Jazbec, Simon. 2009. "The Properties and Applications of Quasicrystals." In Seminar II, university of Ljubljana, Ljubljana. http://mafija.fmf.unilj.si/seminar/files/2009_2010/Quasicrystals.pdf

Kleiner M. and Tichy J. 2014. Acoustics of Small Rooms. London and New York: Taylor \& Francis CRC Press. https://doi.org/10.1201/b16866 
Lam, Y. W. 1999, "A boundary integral formulation for the prediction of acoustic scattering from periodic structures." J. Acoust. Soc. Am. 105(2): 762-769.

https://doi.org/10.1121/1.426267

Lee, H. and T. Sakuma. 2015. "Numerical characterization of acoustic scattering coefficients of one-dimensional periodic surfaces." Applied Acoustics 88: 129-136. https://doi.org/10.1016/j.apacoust.2014.08.014

Lee, H., Y. Tsuchiya, and T. Sakuma. 2018. "Acoustic scattering characteristics of Penrose-tiling-type diffusers." Applied Acoustics 130:168-176.

https://doi.org/10.1016/j.apacoust.2017.08.022

Levine, D. and P. Steinhardt. 1984. "Quasicrystals: a new class of ordered structures." Phys. Rev. Lett. 53: 2477. https://doi.org/10.1103/PhysRevLett.53.2477

Levine, D. and P. Steinhardt. 1986. "Quasicrystals. 1. Definition and structure." Phys. Rev. B 34: 596-616. https://doi.org/10.1103/PhysRevB.34.596

Lord, E.A., S. Ranganathan and U.D. Kulkarni. 2000. "Tilings, coverings, clusters and quasicrystals." Curr. Sci. 78 (1): 64 .

Lu, P. and P. Steinhardt. 2007. "Decagonal and quasicrystalline tilings in medieval Islamic architecture." Science 315:11061110. https://doi.org/10.1126/science.1135491

Maclá, Enrique. 2006. "The role of aperiodic order in science and technology." Reports on Progress in Physics 69(2): 397. https://doi.org/10.1088/0034-4885/69/2/R03

Madison, Alexey E. 2015a "Atomic structure of icosahedral quasicrystals: stacking multiple quasi-unit cells." RSC Adv. 5: 79279-79297. https://doi.org/10.1039/C5RA13874D

Madison, Alexey E. 2015b. "Substitution rules for icosahedral quasicrystals." RSC Adv. 5: 5745-5753. https://doi.org/10.1039/C4RA09524C

Makovicky, E. 1992. "800-Year-Old Pentagonal Tiling from Maragha, Iran, and the New Varieties of a Periodic Tiling it Inspired." In Fivefold Symmetry, ed. I. Hargittai, 67-86. Singapore: World Scientific Publishing Co Pte Ltd. https://doi.org/10.1142/9789814439497_0004

Makovicky, E. and N. Makovicky. 2011. "The first find of dodecagonal quasiperiodic tiling in historical Islamic architecture." J. Appl. Cryst. 44: 569-573.

https://doi.org/10.1107/S0021889811013744
Makovicky, E., F. Rull P'erez and P. Fenoll Hach-Al'ı. 1998. "Decagonal patterns in the islamic ornamental art of Spain and Morocco. Bolet'" In Sociedad Espa nola Mineralog'ıa. 21:107-127.

Martinsons, M., M. Sandbrink and M. Schmiedeberg. 2014. "Colloidal trajectories in two-dimensional lightinduced. quasicrystals with 14-fold symmetry due to phasonic drifts." Acta Physica Polonica A 126: 568. https://doi.org/10.12693/APhysPolA.126.568

Mikhael, J., M. Schmiedeberg, S. Rausch, J. Roth, H. Stark and C. Bechinger. 2010. "Proliferation of anomalous symmetries in colloidal monolayers subjected to quasiperiodic light fields." Proc Natl Acad Sci USA 107:7214. https://doi.org/10.1073/pnas.0913051107

Ning, J. F. and G.P. Zhao. 2017. "A fractal study of sound propagation characteristics in roughened porous materials." Wave Motion 68: 190-201.

https://doi.org/10.1016/j.wavemoti.2016.09.013

Penrose, R. 1974. "The role of aesthetics in pure and applied mathematical research." Bull. Inst. Math. Appl. 10: 266-271.

Qian, Z. W. 2001. "Wave scattering on a fractal surface.". J. Acoust. Soc. Am. 107: 260-262.

https://doi.org/10.1121/1.428302

Rigby, J. 2005. "A Turkish interlacing pattern and the golden ratio." Math. School. 34: 16-24.

Roichman, Y. and D. G. Grier .2005. "Holographic assembly of quasicrystalline photonic heterostructures." Optics Express 13: 5434-5439. https://doi.org/10.1364/OPEX.13.005434

Saltzman, P. W. 2008. "Quasi-periodicity in Islamic ornamental design". In Nexus VII: Architecture and Mathematics Conference Series, ed. Kim Williams. 153168. Torino: Kim Williams Books.

Schmiedeberg, M., C.V. Achim, J. Hielscher, S. C. Kapfer and H. Löwen. 2017. "Dislocation-free growth of quasicrystals from two seeds due to additional phasonic degrees of freedom." Physical Review E 96: 012602.

Schmiedeberg, M. and H. Stark. 2012. "Comparing lightinduced colloidal quasicrystals with different rotational symmetries." J Phys Condens Matter 24: 284101. https://doi.org/10.1088/0953-8984/24/28/284101

Schroeder, M. R. 1975. "Diffuse sound reflection by maximum- length sequences." The Journal of the 
Acoustical Society of America 57(1):149-150.

https://doi.org/10.1121/1.380425

Schroeder, M. 1975. "Binaural dissimilarity and optimum ceilings for concert halls: more lateral sound diffusion." The Journal of the Acoustical Society of America 65: 95863. https://doi.org/10.1121/1.382601

Shechtman, D., I. Blech, D. Gratias and J.W. Cahn. 1984.

"Metallic phase with long range orientational order and no translation symmetry." Phys. Rev. Lett. 53(20): 951-1954.

https://doi.org/10.1103/PhysRevLett.53.1951

Socolar, J., P. Steinhardt and D. Levine. 1985.

"Quasicrystals with arbitrary orientational symmetry."

Phys. Rev. B 32: 5547-5550.

https://doi.org/10.1103/PhysRevB.32.5547

Steurer, W. and D. S. Widmer. 2007. "Photonic and phononic quasicrystals." J Phys D: Appl Phys 40:R229R247. https://doi.org/10.1088/0022-3727/40/13/R01

Yamamoto, Akiji and Hiroyuki Takakura. 2008. "Recent development of quasicrystallography." In Quasicrystals, ed. by T. Fujiwara and Y. Ishii, 11-47. Amsterdam: Elsevier. https://doi.org/10.1016/S1570-002X(08)80017-1

Zhu, Y., Fan, X., Liang, B., Cheng, J. and Jing, Y. 2017. "Ultrathin Acoustic Metasurface-Based Schroeder Diffuser." Phys. Rev. X 7: 021034.

https://doi.org/10.1103/PhysRevX.7.021034 